\title{
Subjective survival expectations and observed survival: How consistent are they?
}

\author{
Alberto Palloni and Beatriz Novak*
}

\begin{abstract}
In this paper, we use new models to convert subjective expectations elicited from individual responses into conditional survival functions. We also estimate the effects of individual characteristics and assess the impact of health shocks on individual updates of subjective expectations. We use Health and Retirement Study (HRS) data from 1992 to 2006. By and large, our results confirm past empirical findings, but also identify patterns not documented in previous research. We show that the subjective probabilities are remarkably close to the results of actual life tables constructed from observed data, that whites underestimate their survival chances more than blacks, that women underestimate their survival chances more than men, and that the subjective underestimation of conditional survival increases with age in all population subgroups. We find significant differences in the survival outlooks of the original HRS cohort and a more recent HRS cohort (1992 versus 2004). These differences persist after introducing suitable controls. The observed mortality differentials between smokers and non-smokers, obese and non-obese individuals, and high-education and low-education groups are quite close to those of these subgroups' subjective survival expectations. Finally, we find large updating effects that result from recent health shocks on subjective expectations.
\end{abstract}

\section{Introduction}

Subjective survival expectations are responses to questions about the probabilities of surviving to selected target ages that respondents could attain in the future. The strategies used to elicit probabilistic appraisals from individuals are quite diverse, but the standard tools are based on variants of a question such as this: "Using

* Alberto Palloni, Center for Demography of Health and Aging, University of Wisconsin-Madison, 4434 Sewell Social Sciences, 1180 Observatory Drive, Madison, Wisconsin 53706-1393, U.S.A. Email: palloni@ssc.wisc.edu

Beatriz Novak, Center for Demographic, Urban and Environmental Studies, El Colegio de Mexico 
a number between 0 and 1 , where 0 is lowest and 1 is highest, what would you say is the probability that you will survive to age $X$ (or $Y$ more years)?" Work on survival expectations is relatively new, and is part of a larger literature on individual expectations (Manski 2004). The interest in this topic has been growing rapidly as researchers uncover patterns and varying degrees of consistency of subjective expectations with individual objective health status and changes thereof (Liu et al. 2007), past and current health-related behaviors (Falba and Busch 2005; Khwaja et al. 2006 and 2007; Scott-Sheldon et al. 2010), experiences of health shocks, and individual self-reported health (Smith et al. 2001a).

This paper has two modest goals. First, we seek to evaluate the degree of consistency between conditional survival probabilities computed from subjective survival expectations and conditional survival probabilities from observed life tables in two cohorts of older adults from the Health and Retirement Study (HRS). To do so, we employ models and strategies that partially resolve well-known problems of subjective expectation data. Second, we estimate the effects of individual traits (obesity), behaviors (smoking), and experiences with health shocks on subjective survival probabilities, and assess how these effects vary by age, race, and gender.

In this paper, we seek to confirm empirical results obtained by past research, and to introduce a handful of new ideas and findings. First, we propose semi-parametric, relational models that capture robustly the effects of individual traits, behaviors, and health shocks on the formation and updating of subjective expectations. Second, we perform extensive - albeit tedious - sensitivity analyses to assess the magnitude of the estimates' uncertainty that is attributable to a recurrent problem of subjective expectations; namely, the large shares of focal ${ }^{1}$ and inconsistent responses. ${ }^{2}$ Third, we identify and account for the phenomenon whereby older individuals tend to underestimate their survival chances (relative to their objective chances) by a larger margin than younger individuals. Finally, we add new empirical evidence to the literature about the direction and the magnitude of the updating of individual expectations as a reaction to recent health shocks (Khwaja et al. 2006; Liu et al. 2007; Smith et al. 2001a and 2001b).

The outline of the paper is as follows. In section 2, we review selected empirical findings. In section 3 , we describe the data, including the advantages and disadvantages of our dataset. In sections 4 and 5, we introduce definitions, describe the models, and review the results. We present our conclusions in the final section.

\footnotetext{
1 A focal response is an answer given to a question in which the respondent is asked about his or her beliefs regarding the probability of the occurrence of any given future event that corresponds to a probability equal to zero, one-half, or one (Hurd and McGarry 1995).

2 In this paper, inconsistency occurs when the implied probability of surviving to age $y$ is lower or equal to the implied probability of surviving to age $x, x>y$.
} 


\section{Subjective survival expectations, health, and mortality}

While self-rated health status (SRH) reflects a combination of specific information and knowledge about recent or current health problems, physical functioning, and health-related behaviors (Quesnel-Vallée 2007), subjective survival expectations may capture some richer dimension that is unlikely to be included in self-rated health status (Popham and Michell 2007). It has been posited that these expectations reflect information about genetic or hereditary susceptibilities (Balia 2007, Hurd and McGarry 1995; Perozek 2008; Roebuck Bulanda and Zhang 2009); knowledge of the health conditions of parents, siblings, or other kin (Zick et al. 2014; van Doorn and Kasl 1998); assessments of past or current exposures to environmental and behavioral risk factors not captured by standard surveys questionnaires (Perozek 2008); and experiences of health trajectories over time (Benítez-Silva and Ni 2008).

There is abundant evidence showing that self-rated health status is a consistently robust independent predictor of mortality, even after accounting for relevant covariates, such as objective health status indicators (Idler and Benyamini 1997; Singh-Manoux et al. 2007; Young et al. 2010). Since research on subjective survival expectations is a new area of health research, it is not yet widely known that these expectations have been shown in some analyses to be good predictors of individual mortality, even after controlling for socio-demographic factors and healthrelated conditions (Hurd et al. 1999; Hurd and McGarry 2002; Perozek 2008; Smith et al. 2001b). However, these outcomes are not uniformly supported by all empirical findings, and some researchers continue to be skeptical about their power (Elder 2013). More surprisingly, although it has been shown that subjective survival expectations are empirically related to self-rated health, and that these indicators are roughly consistent with each other, there is evidence that survival expectations tap different dimensions of individual health vulnerabilities (Hurd and McGarry 1995; van Doorn and Kasl 1998), and are independent predictors of both individual and aggregate mortality, net of the effects of self-rated health (Siegel et al. 2003; van Doorn and Kasl 1998).

Recent research on subjective survival expectations has identified four important patterns.

First, individual subjective probabilities of survival are roughly consistent with observed (life table) probabilities, exhibit the expected levels of covariation with characteristics such as socioeconomic status and smoking (Hudomiet and Willis 2012; Hurd and McGarry 1995), are roughly consistent with populationbased observed survival patterns, and track remarkably well with observed individual survival trajectories (Smith et al. 2001b; Hurd and McGarry 2002) As Smith and colleagues stated in their study of HRS data, “... [the] evolution of subjective probabilities does appear to include an expectational component that may incorporate unobservable features of personal circumstances that bear on survival to age 75" (Smith et al. 2001b: 1131).

Second, survival expectations track reasonably well with changing mortality conditions. Thus, in some of the most thorough work to date using HRS data, 
Perozek (2008) found that subjective expectations elicited from the 1992 HRS predict the direction of revisions to the Social Security Administration (SSA) life tables between 1992 and 2004. Studies based on the European SHARE data lend support to this observed pattern, and lead to similar inferences (Peracchi and Perotti 2012). However, the degree of accuracy with which subjective expectations "foresee" or track mortality changes is contingent upon and influenced by the age of the individual and the age that is used as a target to elicit expectations. Thus, Elder (2013) found that older HRS respondents became more "pessimistic" regarding their future survival (i.e., they underestimated their objective survival probabilities), and that respondents in the original HRS cohort (1992) were more "optimistic" (i.e., they overestimated their objective survival probabilities) than the cohort recruited in 2004.

Third, empirical research has also uncovered some important discrepancies between subjective expectations and observed experiences. Thus, Hamermesh (1985) showed that individuals underestimate their short-term survival probabilities and overestimate their long-term survival probabilities relative to actuarial life table estimates. Similarly, Elder (2007) found that in the 1992-2004 HRS, the younger respondents (early fifties) were too "pessimistic" about their chances of surviving to young old ages, but that the older respondents (early sixties) were considerably more "optimistic" regarding their chances of surviving to more advanced ages (particularly to age 85 and older) when compared to actuarial estimates ("flatness bias"). A study by Post and Hanewald (2010) found that older individuals were more "optimistic" about their survival prospects (i.e., they overestimated their survival chances), and that, not surprisingly, these subjective expectations translated into more conservative saving behaviors.

Fourth, and finally, it has been suggested many times that subjective survival probabilities are likely to contain information that is not captured by either selfreported health status or objective measures of health limitations (Popham and Mitchell 2007). Hurd and McGarry (2002) found that self-rated health status and subjective survival expectations among individuals aged 46-65 were independently associated with individual mortality experiences between the first and second $H R S$ waves, even after accounting for effects of multiple confounders, including socio-demographic characteristics and objective measures of health. This result is corroborated in a study that used information from the first wave of the Assets and Health Dynamics among the Oldest Old (AHEAD) study (individuals aged 70 and older) (Siegel et al. 2003). Additional evidence regarding the unique dimensions tapped by subjective expectations comes from the Australian Longitudinal Study of Aging (ALSA), in which researchers found that individuals' subjective survival expectations and mortality risk estimates were related to parental longevity (van Doorn and Kasl. 1998).

Although the above findings are roughly consistent and recurrent, there are also some salient conflicting results. This should not be surprising in a new research area in which there are unresolved disagreements about concepts, measurements and models, and the utilization of data sources that are not always well harmonized. 
In this paper, we examine empirical evidence pertaining to the first (consistency) and fourth (responsiveness to individual health experiences) patterns outlined above. We do so by using strategies that partially circumvent three problems that are commonly encountered: (a) how to define robust models that can translate subjective expectations into standard conditional survival patterns that are comparable to the observed patterns; (b) how to treat "ambiguous" subjective expectations (focal and inconsistent responses); and (c) how to identify formalized protocols for making statistical inferences about the effects of individual traits, behaviors, exogenous changes, and time.

In the following, we address all three problems. First, we formulate robust models that can translate subjective survival expectations into predictions of mortality risks that are comparable to objective mortality rates. These models are flexible and easily estimated, and their relative performance is readily evaluated. Second, we assess the sensitivity of empirical estimates to the treatment of imprecise information; e.g., the ubiquitous and pervasive presence of focal and inconsistent responses. Although all researchers are well aware that if left unresolved, these responses engender problems that could lead to flawed inferences (Bassett and Lumsdaine 2001; Gan et al. 2005; Kuwaja et al. 2007; Lillard and Willis 2001; Lumsdaine and Potter van Loon 2012), not all published work on the subject has investigated the parameter uncertainty these responses could generate. Finally, we use variants of generalized accelerated failure time models to evaluate the impact of individuals' characteristics, behaviors, and experiences with exogenous shocks on the formation of subjective survival expectations.

\section{The data}

\subsection{The health and retirement study}

The data used in this study are drawn from the Health and Retirement Study (HRS). ${ }^{3}$ The HRS is a longitudinal survey designed to gather information on individuals in the U.S. from pre-retirement into retirement. The first wave's (1992) target population included individuals born between 1931 and 1941 who were living in households, and the spouses or partners of these individuals, regardless of their ages. Out of the 15,497 individuals who were eligible to be interviewed in 1992, 12,654 respondents were actually interviewed. Since then, the individuals in this initial cohort have been re-interviewed every two years. The entire survey consists of five birth cohorts who have been incorporated into the study over time. In the present study, we examine data from the first, fourth, and fifth HRS cohorts. The first cohort is the initial HRS cohort. The fourth cohort, the War Baby (WB) cohort, consists of 2,529 individuals who were born between 1942 and 1947.

3 For a detailed description of the HRS, refer to: Juster and Suzman (1995). 
These respondents were first interviewed in 1998, and every two years thereafter. Finally, the fifth cohort, the Early Baby Boomer (EBB) cohort, consists of 3,340 individuals who were born between 1948 and 1953. These respondents were first interviewed in 2004, and every two years thereafter. Information by racial groups comes from oversamples of Hispanics, blacks, and Florida residents. ${ }^{4}$ Finally, the HRS also includes information on the survival status of the respondents, and linked information about decedents from the National Death Index. We use data from the first eight HRS waves (1992-2006). Tables 1 and 2 display the pertinent descriptive statistics for the sample cohorts we use in this paper; namely, respondents who were aged 50 to 61 in 1992 and in 2004.

\subsection{Subjective expectations in the HRS}

Since 1992, the HRS has included a number of questions designed to elicit respondents' subjective expectations - or, more precisely, subjective probability distributions - regarding a range of future events. In 1992, the questions on subjective survival probabilities were as follows: "Using any number from zero to ten, where ' 0 ' equals absolutely no chance and ' 10 ' equals absolutely certain... What do you think are the chances that you will live to be 75 or more?" "And how about the chances that you will live to be 85 or more?" From 1994 onward the questions were reworded as follows: "Please answer the questions in terms of percent chance. Percent chance must be a number from 0 to 100 , where ' 0 ' means there is absolutely no chance, and ' 100 ' means that it is absolutely certain." In addition to including this change, the 1994 HRS wave was the first wave that restricted the age at which the first of the above-mentioned questions (survival to age 75) was posed to individuals aged 65 and younger, and the age at which the second question was posed to individuals aged 75 and younger. This format was maintained for the 1996 and 1998 HRS waves. In 2000, the question took on the following format: "How sure are you that you are going to live to be... 75 years or more (if the respondent's age is less than or equal to 65 years); 80 years or more (age 69 years or less); 85 years or more (age between 70 and 74 years); 90 years or more (age between 75 and 79 years); 95 years or more (age is 80 and 84 years); and 100 years or more (age between 85 and 90 years)?" As before, zero represented "Absolutely no chance" and 100 represented "Absolutely certain." The subjective survival questions were not posed to proxies.

\footnotetext{
4 The present study uses the RAND version of the HRS: RAND HRS Data File Version "H" (http://hrsonline.isr.umich.edu/modules/meta/rand/index.html).
} 


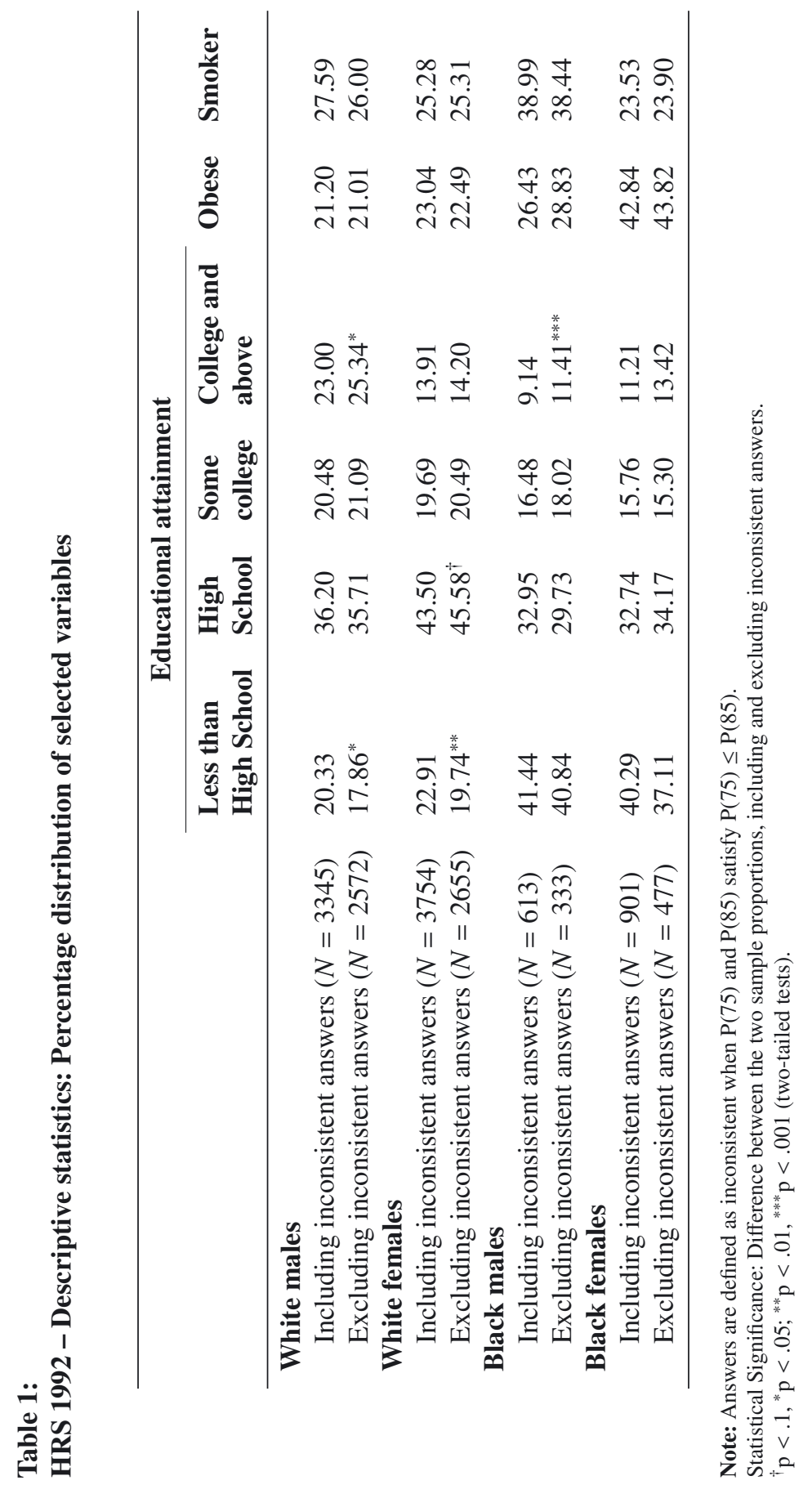




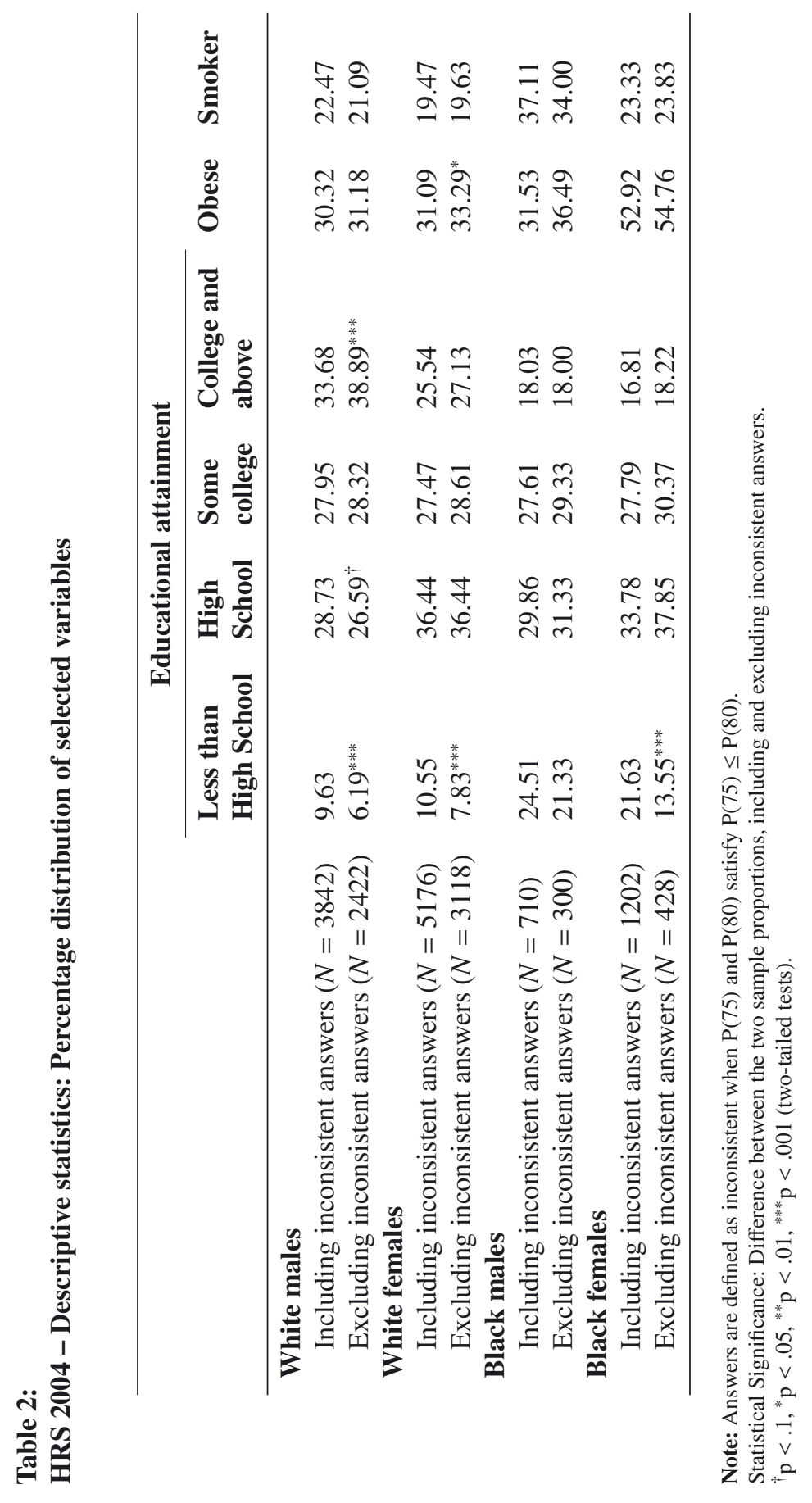


Figure 1:

Subjective survival to age 75 . Focal responses $(0,50$, and 100$)$ : percentages by gender and race - HRS 1992

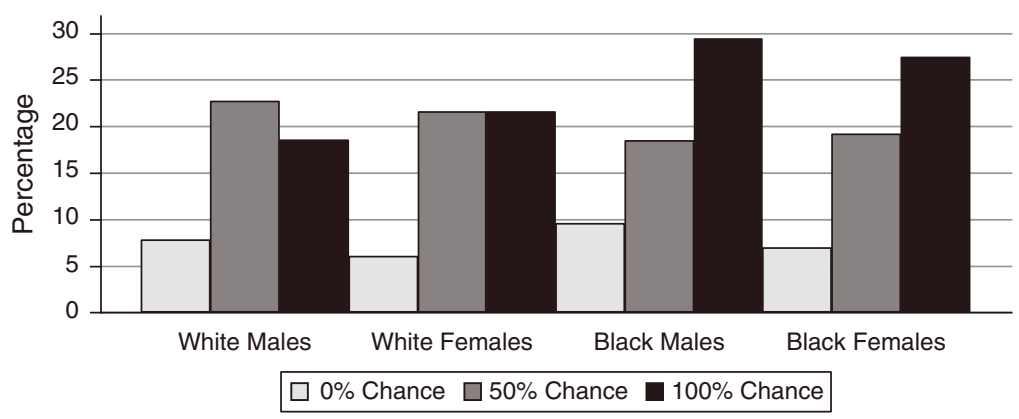

Figure 2:

Subjective survival to age 85 . Focal responses $(0,50$, and 100): percentages by gender and race - HRS 1992

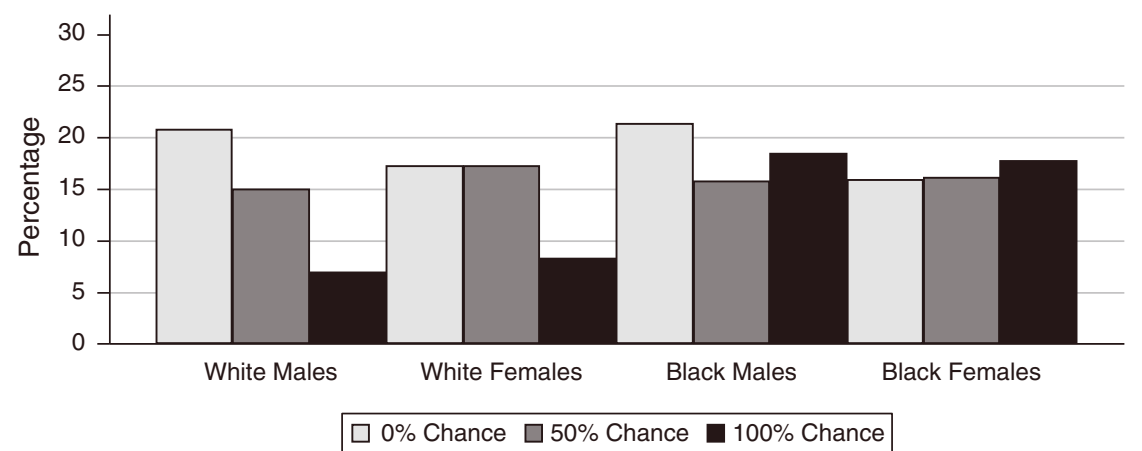

\subsection{Focal and inconsistent responses in the HRS}

Of the 9,746 individuals (52.97\% of whom were women) aged 50-61 who were interviewed in 1992, 9,049 answered the questions on subjective survival to ages 75 and 85. As of 2006, 1,511 deaths were registered among the individuals in this population, and a total of 828 individuals were lost to follow-up. Statistics summarizing selected variables are in Tables 1 and 2. Figures 1 and 2 show the gender- and race-specific prevalence of focal responses given in 1992 to the 
questions on subjective survival to ages 75 and 85 , respectively. ${ }^{5}$ These figures show that focal answers were common. Across all gender and race groups, the heaping on answers reflecting a " $0 \%$ chance" of surviving ranged from $7.8 \%$ to $9.5 \%$ for age 75 and from $15.9 \%$ to $21.3 \%$ for age 85 . The heaping on answers reflecting a " $50 \%$ chance" of surviving ranged from $18.4 \%$ to $22.7 \%$ for age 75 and from $15.0 \%$ to $17.3 \%$ for age 85 . Finally, the heaping on answers reflecting a "100\% chance" of surviving ranged from $18.5 \%$ to $29.4 \%$ for age 75 and from $6.9 \%$ to $18.5 \%$ for age 85 .

The noise arising from heaping or focal responses was made worse by the inconsistency that occurred when the implied probability of surviving to age 75 was lower or equal to the implied probability of surviving to age 85 (1992 HRS) or to age 80 (2004 HRS). Figure 3 displays the prevalence of the inconsistent responses by gender, by race, and by whether the response was focal (heaped). Unsurprisingly, we find that the largest fraction of inconsistent responses was among the focal answers, and was dominated by cases with an elicited probability of surviving to age 75 that was equal to the elicited probability of surviving to age 85 (or to age 80 , depending on the HRS wave). The percentage of inconsistent responses resulting from the elicited probabilities of surviving to age 75 that equaled the elicited probabilities of surviving to age 85 ranged from $17.7 \%$ among white males to $33.6 \%$ among black males.

Although the educational levels of the individuals who offered inconsistent and/or focal responses differed from those of the individuals who offered consistent and non-focal responses, these differences were small. In particular, the educational distribution among whites with appropriate responses was slightly slanted toward higher educational attainment (see Tables 1 and 2). ${ }^{6}$

\section{Models and estimation}

\subsection{Translation of subjective expectations into conditional survival probabilities}

We use the answers to the 1992 questions eliciting the individual probabilities of surviving from age $x$ to ages 75 and 85. Each individual response is an observation of the (subjective) conditional probability of surviving from age $x$ to ages 75 and $85, S_{x}(75)$ and $S_{x}(85)$. We assume that these observables are synthetic outcomes generated by a latent individual assessment of mortality risks, and that there is intra-individual homogeneity in the assessments, such that the set of pairs $\left(S_{x}(75)\right.$,

\footnotetext{
5 Figures A.1 and A.2 in the appendix show the gender- and race-specific prevalence of focal responses given in 2004 to the questions on subjective survival to ages 75 and 80, respectively.

6 Differentials in the focal and the inconsistent responses by birth cohorts, obesity status, and smoking behavior are quite small, and are not shown here.
} 
Figure 3:

Subjective survival to ages 75 and 85 . Focal responses $(0,50$, and 100): Inconsistent responses percentages by gender and race - HRS 1992

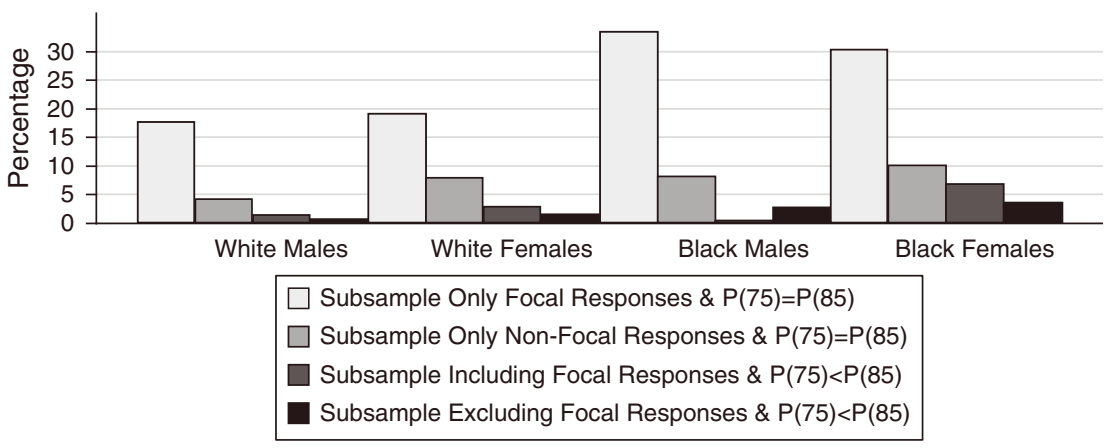

$\left.S_{x}(85)\right)$ observations is consistent with only one pattern of age-specific mortality risks. ${ }^{7}$ We employ four different mortality models, Gompertz, Weibull, logistic, and log-logistic, and estimate the parameters via standard Non-Linear Least Squares routines. ${ }^{8}$ We use the same models to represent the actual mortality experiences observed in the HRS sample and in the official U.S life tables. (1992). ${ }^{9}$ Figures 4 and 5 show the survival curves for all four functions. The logistic and log-logistic models are the best fits, and even though the Gompertz model does not fit as well, it performs well enough. This is in contrast to the much worse performance of the Weilbull function.

Tables 3 to 6 display the parameters for the Gompertz, logistic, Weibull, and loglogistic models estimated from the observed HRS follow-up mortality experiences and from the subjective survival expectations to ages 75 and $85 .{ }^{10}$ The figures in Tables 3 and 4 are computed from the entire sample of respondents who answered questions regarding expectations, whereas the figures in Tables 5 and 6 only employ the sample of consistent responses (but including focal responses). Not surprisingly,

\footnotetext{
7 This approach is different from those implemented in some previous studies. We use expectations associated with two ages as if they were influenced by a common assessment of mortality risks, rather than as two different expressions of different evaluations of the future.

8 When the focal responses were equal to zero or one, we replaced the survival probabilities with 0.001 or 0.999 respectively.

9 In the following we use the HRS observed mortality experiences as a benchmark for comparison. We include comparisons with functions and parameters computed from the U.S. life tables only in some tables and figures. Since, apart from sampling errors, the HRS mortality experiences reproduce the U.S. life tables quite well, the inclusion of all three set of estimates is somewhat redundant.

10 All standard errors are estimated with corrections for clustering, since each individual contributes two observations corresponding to two subjective survival expectations.
} 


\section{Figure 4:}

White men's and women's survival functions - U.S. 1992 life tables, Kaplan-Meier, Gompertz, logistic, Weibull, and log-logistic model estimates for the observed 14-year HRS mortality (1992 to 2006)
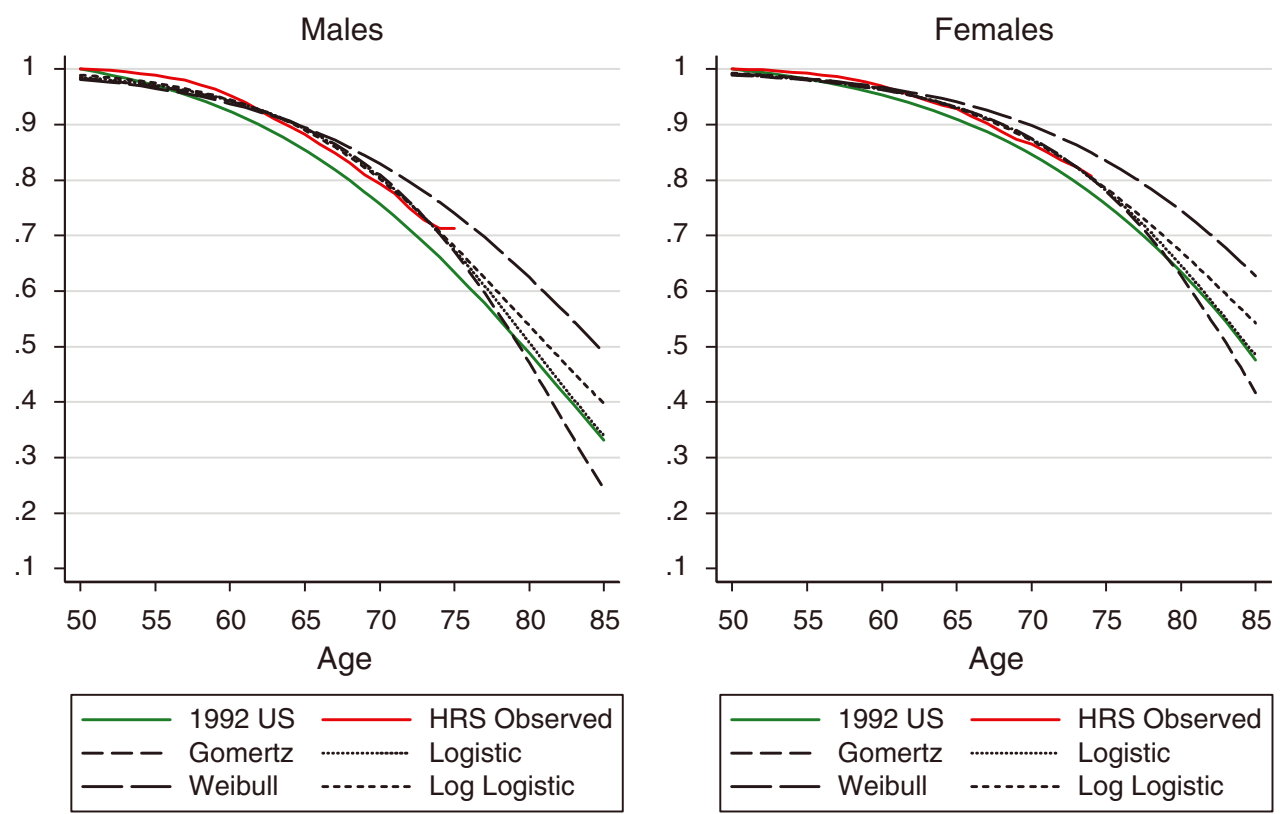

the results suggest that the fit $\left(\mathrm{R}^{2}\right)$ of subjective probabilities is better in the more restricted sample. Importantly, however, the tables also show that the parameter estimates based on the observed experiences are similar across the two subsamples, which suggests that the aggregate mortality experience, at least, is not associated with propensities for inconsistent responses.

In the unrestricted sample (Tables 3 and 4), the main differences between objective and subjective survival are seen in the slope of the mortality curves (the ancillary parameter of the Gompertz, logistic, Weibull, and log-logistic functions): by and large, the age-dependent increase in mortality rates is more than twice as fast in the observed data than in the subjective data. This is because the less exclusionary sample includes pairs of observations $S_{x}(75)$ and $S_{x}(85)$ that imply survival curves with implausibly low slopes (due to inconsistent responses). Indeed, at least for the Gompertz and logistic functions, the contrast vanishes when using the more restrictive sample (Tables 5 and 6) that ignores inconsistent responses.

Although the above result may be unsurprising, a warning should be issued: it is not possible to obtain reasonable estimates of mortality parameters from information on subjective survival expectations that includes inconsistent responses. This is important, as it is common practice to use the subjective expectations for 
Figure 5:

Black men's and women's survival functions - U.S. 1992 life tables, Kaplan-Meier, Gompertz, logistic, Weibull, and log-logistic model estimates for the observed 14-year HRS mortality (1992 to 2006)
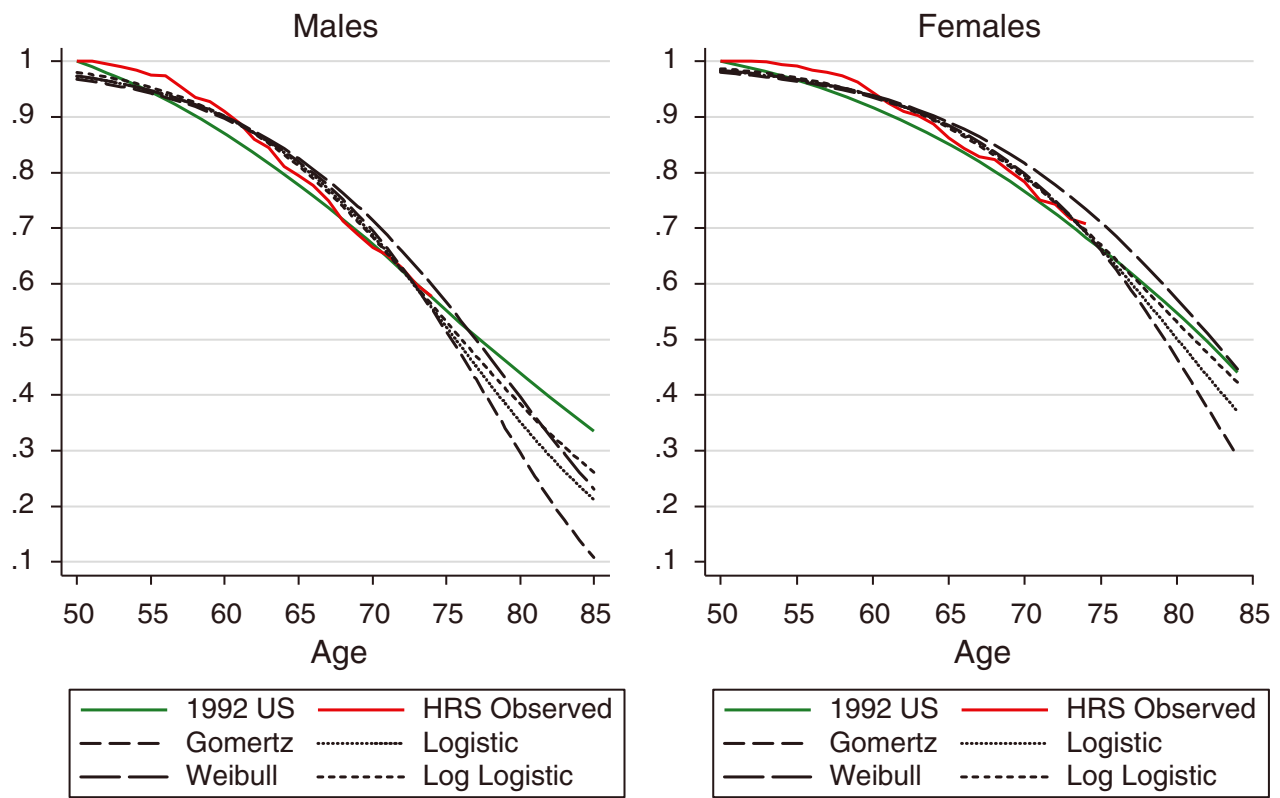

each target age separately, while ignoring the fact that if these expectations are considered jointly, they cannot refer to plausible survival curves. ${ }^{11}$

Figures 6 and 7 show, for each race and gender, the non-parametric Kaplan-Meier survival function for the observed 14-year HRS mortality table (1992 to 2006), the 1992 U.S. mortality life table, and the Gompertz, logistic, Weibull, and log-logistic models fitted to the subjective expectations using the restricted sample. To facilitate comparisons, Tables 7 and 8 in the text display the values of life expectancy at ages 50,60 , and 70 that are implied by each of the fitted models in each group, as well as those from the 1992-2006 HRS mortality experiences and the U.S. 1992 life tables.

While the results from the restricted sample in Tables 5 and 6 show that both the Gompertz and the logistic model represent the mortality trajectories observed in the HRS quite well, the predicted Gompertz survival functions (not shown) fall off with age much more steeply than the observed trajectories; a limitation that the logistic function does not share. This result, which indicates that the logistic model

11 The warning applies only if one is interested in contrasting objective and subjective life tables. It is unnecessary if one is investigating the nature of subjective appraisals by themselves. 


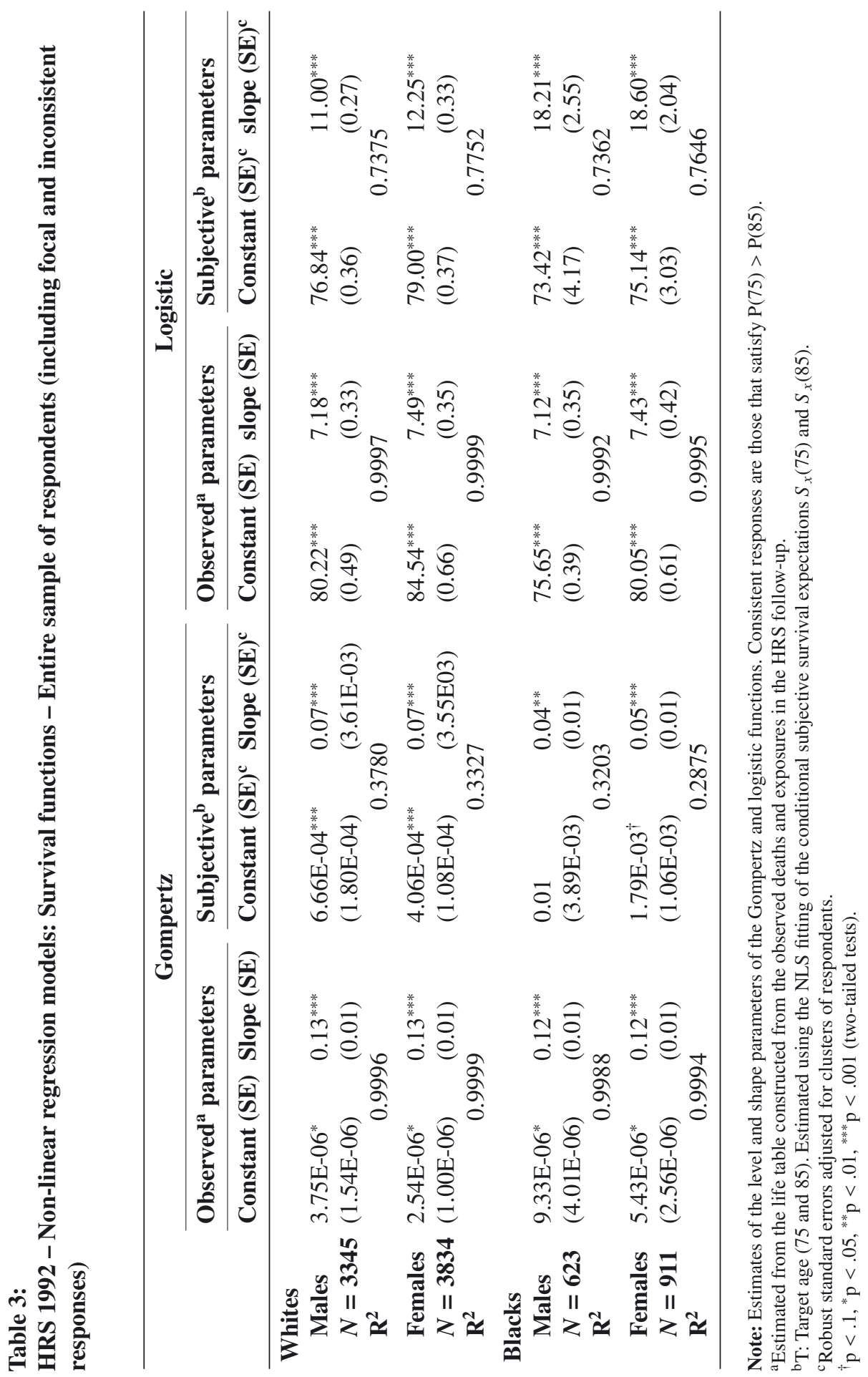




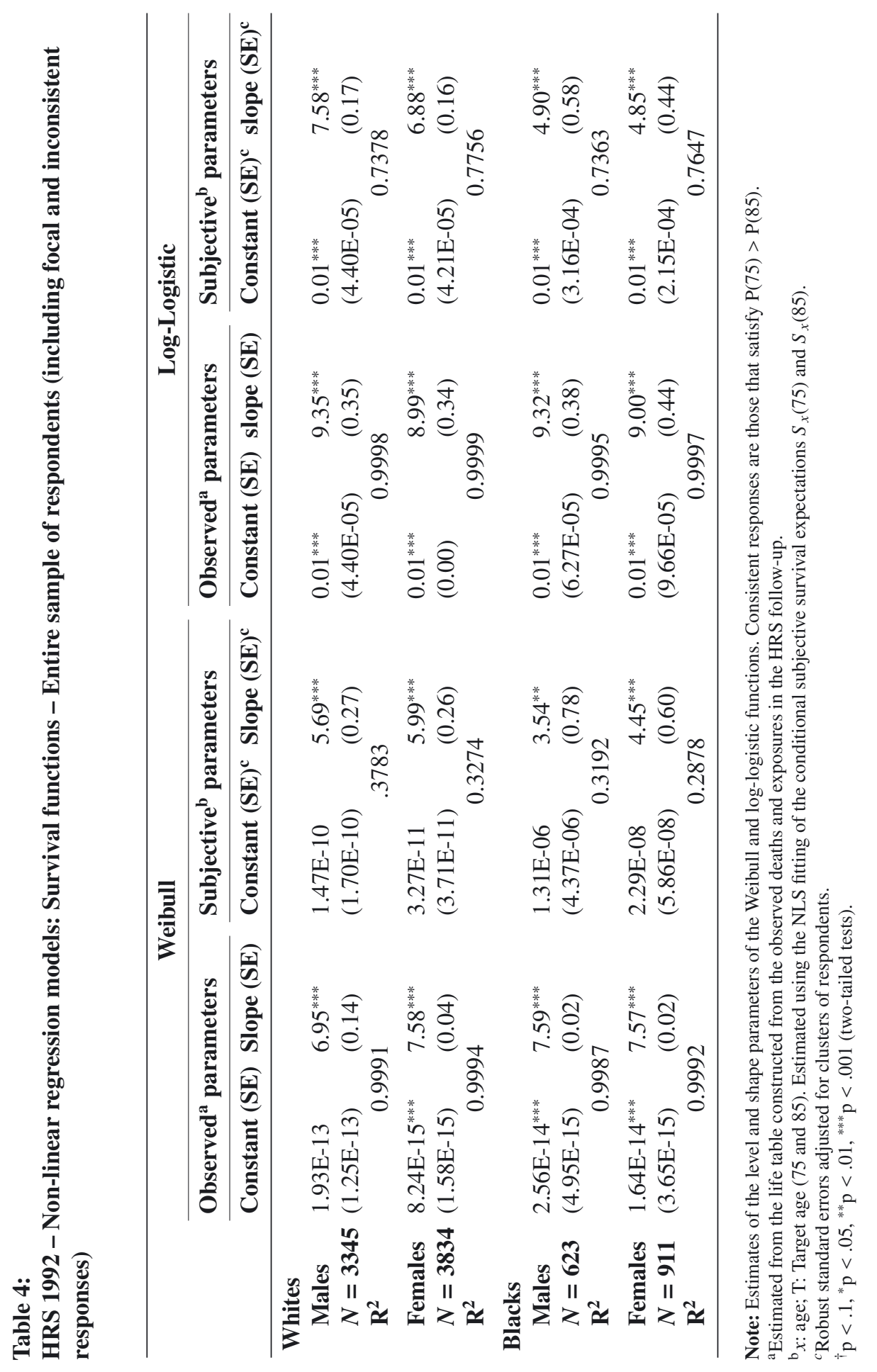




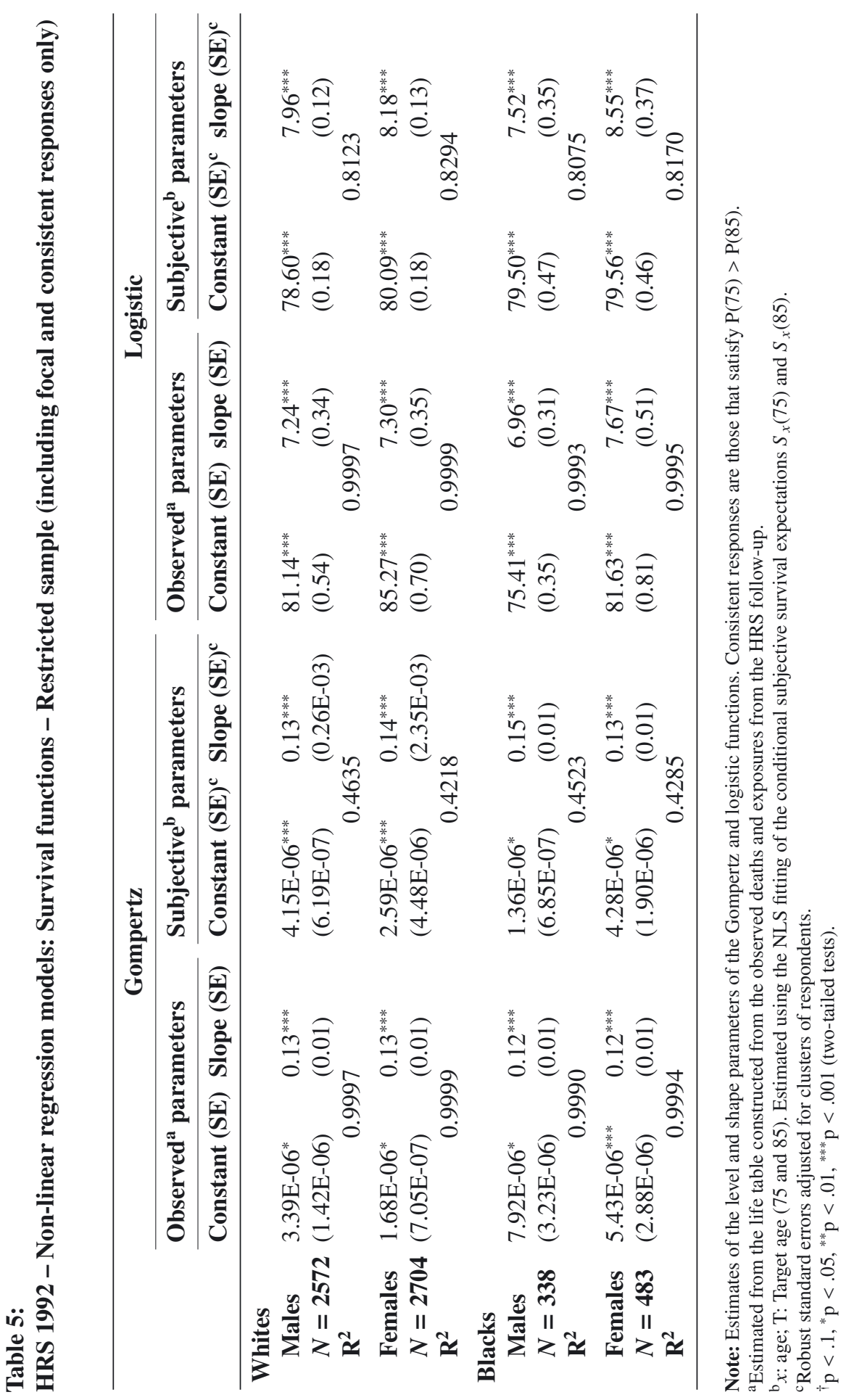




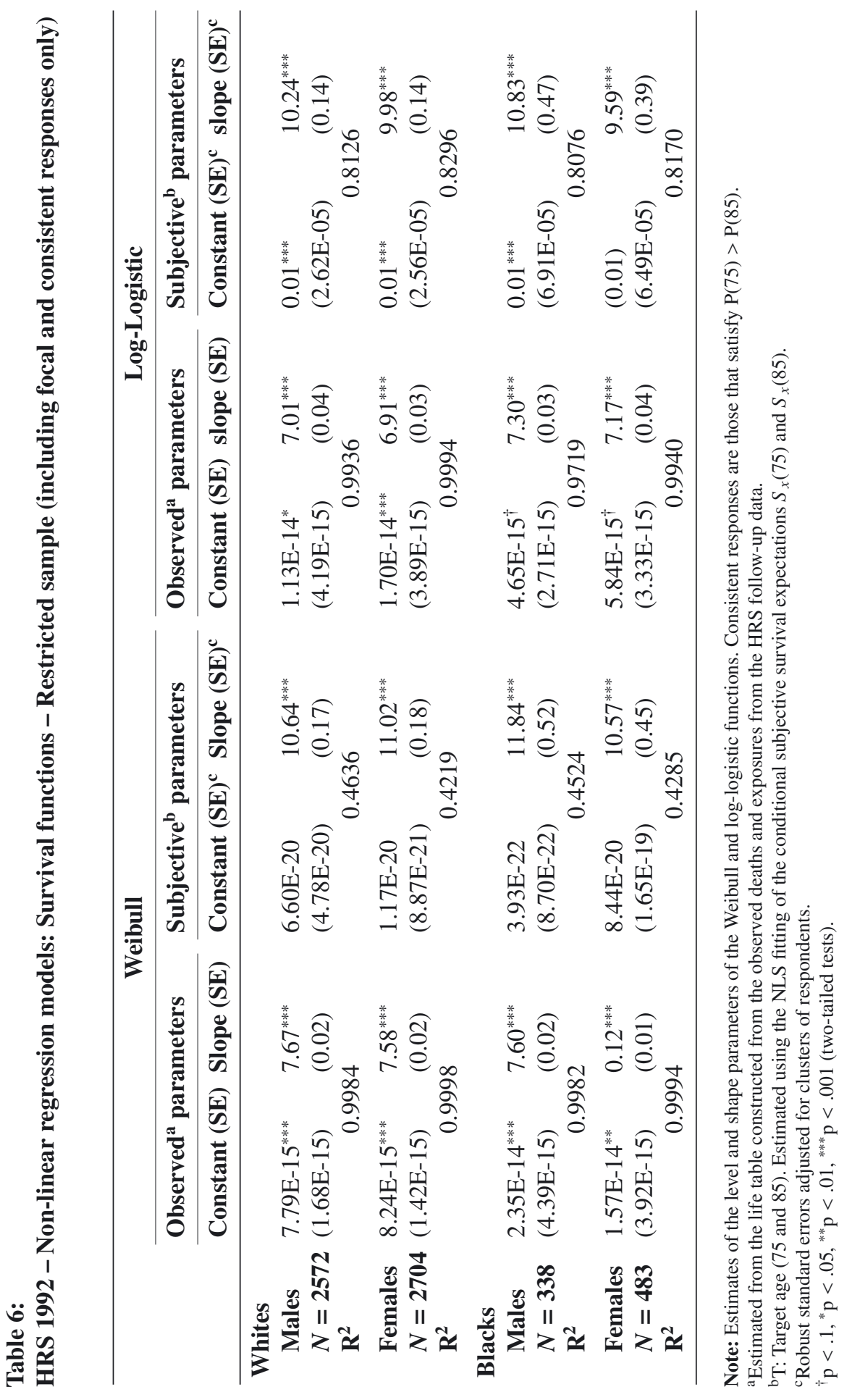


Figure 6:

White men's and women's survival functions - U.S. 1992 life tables, Kaplan-Meier for the observed 14-year HRS mortality (1992 to 2006), and Gompertz, logistic, Weibull, and log-logistic model estimates for subjective survival expectations expressed in 1992 by respondents in the restricted samples
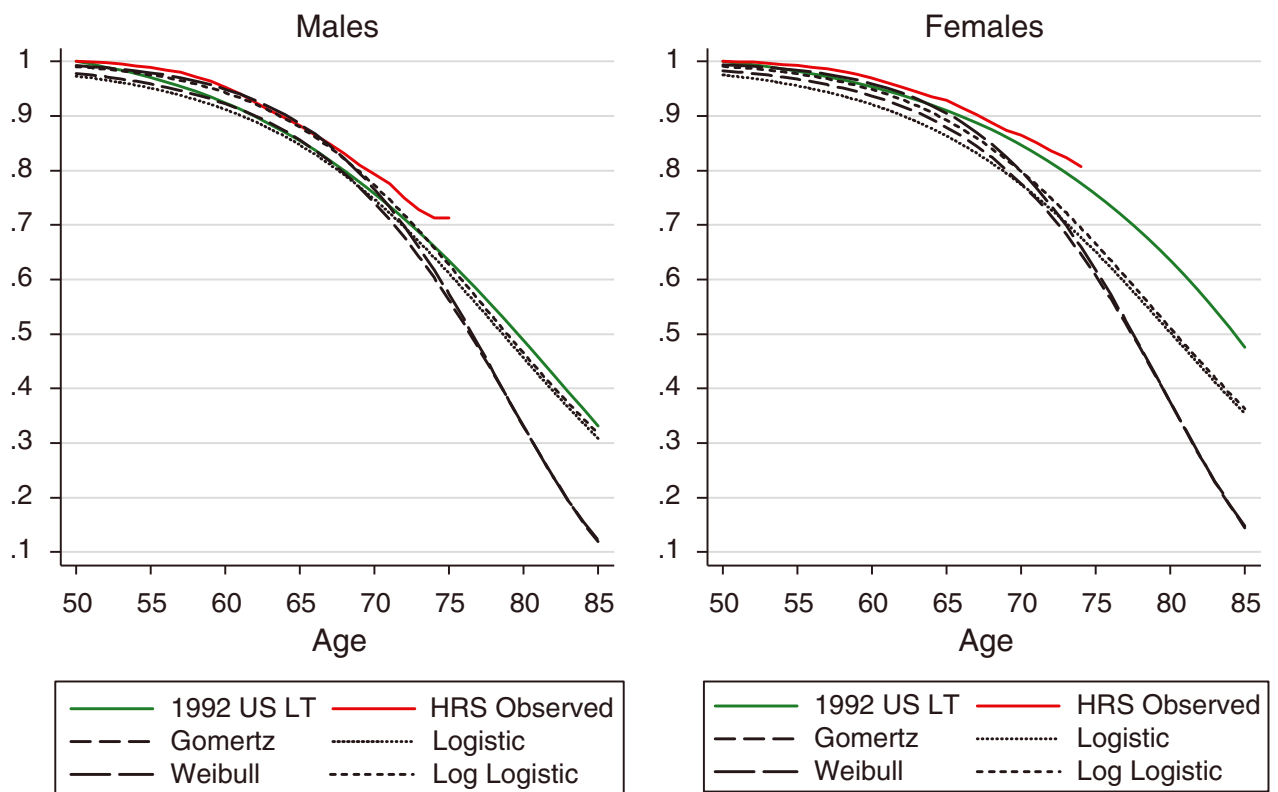

has an edge over the Gompertz model, is in line with recent empirical research on old-age mortality in high-income countries (Horiuchi and Wilmoth 1998; Kannisto et al. 1994; Robine and Vaupel 2001). To ensure consistency with the most recent literature on the subject, we will focus only on the results from the best fitting logistic model.

Tables 7 and 8 reveal three features of the relationship between subjective expectations and experiences of mortality. First, the estimates of life expectancy at ages 50,60, and 70 from all three sources in all four groups are closely clustered, but the differences between the estimates based on subjective expectations and the HRS and/or the U.S. experiences are slightly larger among white women and black men. Among black men, the subjective expectations yield residual expectancies at age 50 that are 4.0 years higher than the HRS observed life table estimates, whereas the black women's estimates are 2.0 years lower than these values. Among whites, the estimates based on subjective expectations are 2.4 years lower for men and 5.0 years lower for women. Thus, white men and women are "pessimistic," whereas black men are quite "optimistic." 
Figure 7:

Black men's and women's survival functions - U.S. 1992 life tables, Kaplan-Meier for the observed 14-year HRS mortality (1992 to 2006), and Gompertz, logistic, Weibull, and log-logistic model estimates for subjective survival expectations expressed in 1992 by respondents in the restricted samples
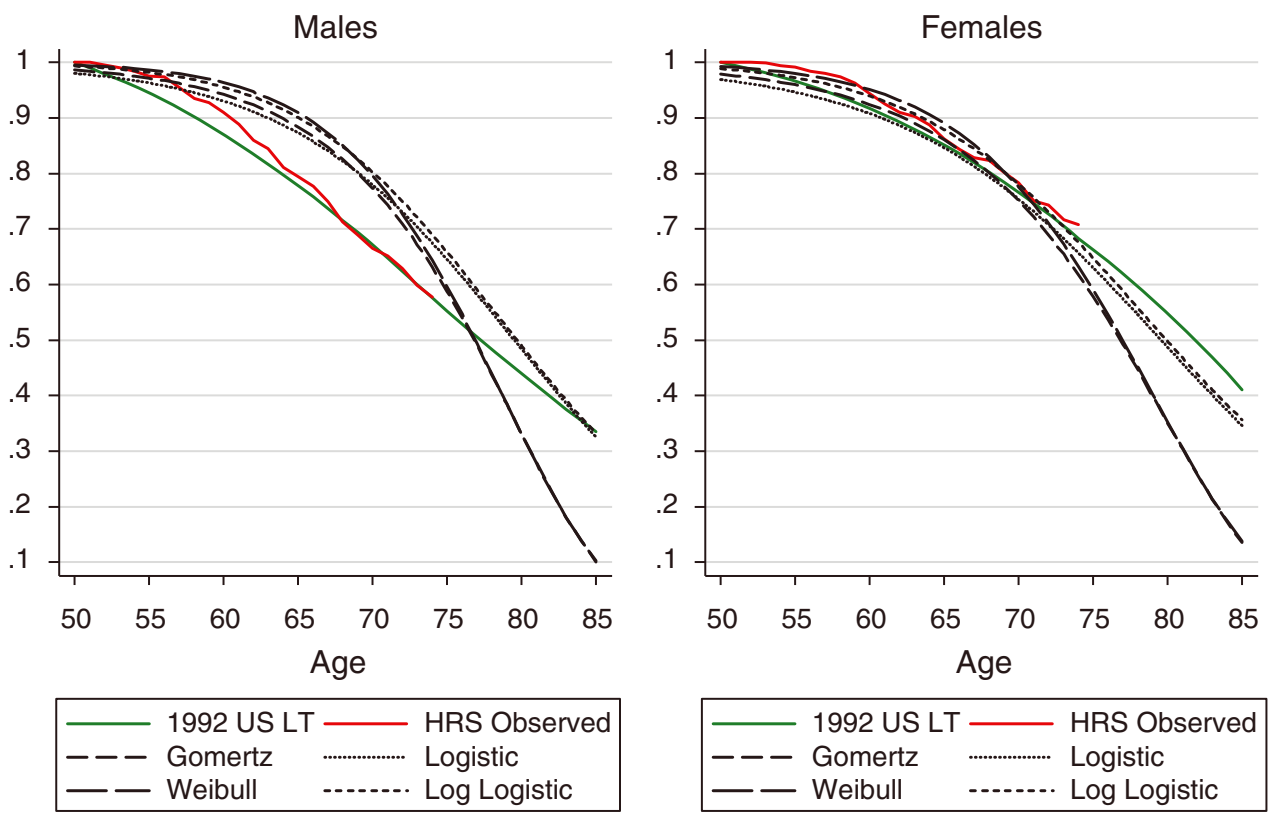

Second, the tables also suggest that subjective expectations reproduce the observed direction of gender disparities only among whites, and the observed direction of race differentials only among women. Furthermore, when the direction of the differentials from the subjective expectations is the same as the observed differentials, the magnitude of the former is less than half that of the latter.

The magnitude of the differences between the estimates from survival expectations and observed mortality is as large, or slightly larger, than the magnitude of the differences between the mortality experiences from the HRS and from the U.S. life tables.

\subsection{Sensitivity of estimates to focal responses}

As we showed above, the estimates of life table parameters from subjective expectations are sensitive to the inclusion of inconsistent responses. Moreover, a sample that includes inconsistent responses produces implausible estimates of 
Table 7:

Whites - Estimated life expectancies at ages 50, 60, and 70 from Gompertz, logistic, Weibull, and Log-Logistic models (1992 Cohort). Restricted sample

\begin{tabular}{lcccccccc}
\hline & \multicolumn{2}{c}{ Age 50 } & & \multicolumn{2}{c}{ Age 60 } & & \multicolumn{2}{c}{ Age 70 } \\
\cline { 2 - 3 } \cline { 7 - 8 } & Males & Females & & Males & Females & & Males & Females \\
\hline U.S. & 27.1 & 31.9 & & 19.1 & 23.2 & & 12.4 & 15.6 \\
HRS Observed & & & & & & & & \\
$\quad$ Gompertz & 28.6 & 32.3 & & 18.9 & 22.5 & & 9.9 & 13.1 \\
$\quad$ Logistic & 31.2 & 35.3 & & 21.5 & 25.5 & & 12.5 & 16.1 \\
$\quad$ Weibull & 34.8 & 38.3 & & 25.0 & 28.5 & & 15.8 & 19.1 \\
$\quad$ Log-logistic & 34.0 & 39.1 & & 24.2 & 29.3 & & 15.3 & 19.9 \\
Subjective & & & & & & & & \\
$\quad$ Gompertz & 25.0 & 26.0 & & 15.4 & 163 & & 6.9 & 7.6 \\
$\quad$ Logistic & 28.8 & 30.3 & & 19.3 & 20.8 & & 10.9 & 12.2 \\
$\quad$ Weibull & 25.6 & 26.6 & & 15.8 & 16.7 & & 7.1 & 7.8 \\
$\quad$ Log-logistic & 30.2 & 31.7 & & 20.4 & 21.9 & & 11.0 & 13.1 \\
\hline
\end{tabular}

Note: Source, estimates from Tables 3 to 6 . The subjective restricted sample includes focal responses but excludes inconsistent responses.

\section{Table 8:}

Blacks - Estimated life expectancies at ages 50, 60, and 70 from Gompertz, logistic, Weibull, and Log-Logistic models (1992 Cohort). Restricted sample

\begin{tabular}{lcccccccc}
\hline & \multicolumn{2}{c}{ Age 50 } & & \multicolumn{2}{c}{ Age 60 } & & \multicolumn{2}{c}{ Age 70 } \\
\cline { 2 - 3 } \cline { 7 - 8 } & Males & Females & & Males & Females & & Males & Females \\
\hline U.S. & 23.0 & 28.5 & & 16.3 & 20.8 & & 11.0 & 14.3 \\
HRS Observed & & & & & & & & \\
$\quad$ Gompertz & 23.7 & 29.0 & & 14.3 & 19.3 & & 6.1 & 10.4 \\
$\quad$ Logistic & 25.6 & 31.8 & & 16.1 & 22.1 & & 8.0 & 13.2 \\
$\quad$ Weibull & 26.5 & 32.3 & & 17.0 & 22.6 & & 8.7 & 13.6 \\
$\quad$ Log-logistic & 27.2 & 34.8 & & 17.7 & 25.1 & & 9.7 & 16.2 \\
Subjective & & & & & & & & \\
$\quad$ Gompertz & 25.5 & 25.4 & & 15.8 & 15.7 & & 7.0 & 7.3 \\
$\quad$ Logistic & 29.6 & 29.8 & & 20.0 & 20.4 & & 11.4 & 12.0 \\
$\quad$ Weibull & 26.0 & 26.0 & & 16.1 & 16.2 & & 7.1 & 7.4 \\
$\quad$ Log-logistic & 30.8 & 31.4 & & 21.0 & 21.7 & & 12.1 & 12.9 \\
\hline
\end{tabular}

Note: Source, estimates from Tables 3 to 6 . The subjective restricted sample includes focal responses but excludes inconsistent responses. 


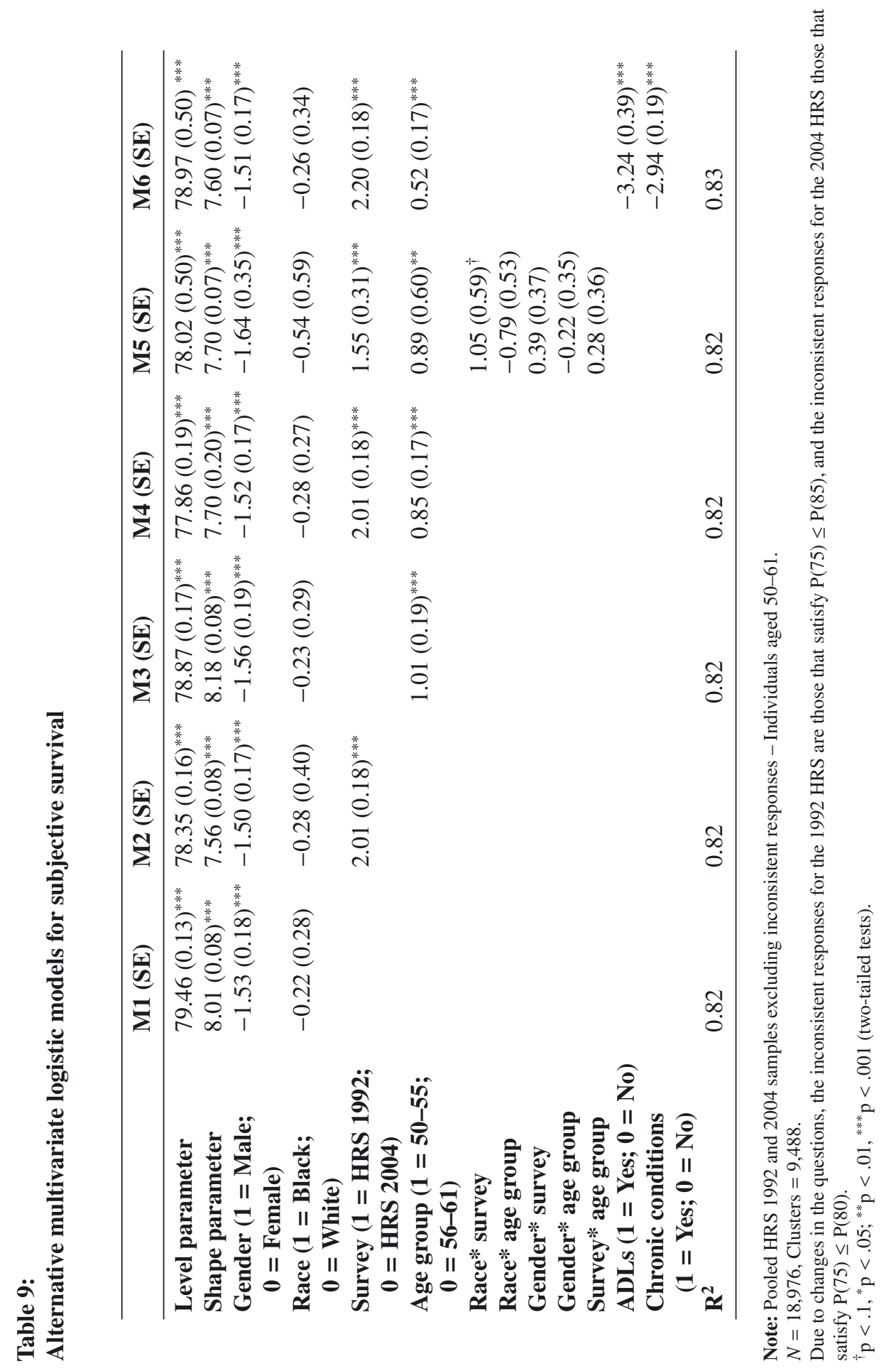


Table 10:

Alternative multivariate logistic models for subjective survival

\begin{tabular}{|c|c|c|}
\hline & M1 (SE) & M2 (SE) \\
\hline Level parameter & $79.40(0.52)^{* * *}$ & $78.08(0.22)^{* * *}$ \\
\hline Shape parameter & $7.49(0.07)^{* * *}$ & $7.49(0.07)^{* * *}$ \\
\hline Gender $(1=$ Male; 0 = Female $)$ & $-1.49(0.18)^{* * *}$ & $-1.78(0.17)^{* * *}$ \\
\hline Race (1 = Black; 0 = White $)$ & $0.07(0.26)$ & $0.48(0.26)$ \\
\hline Smoker $(1=$ Yes; 0 = No $)$ & $-2.69(0.20)^{* * *}$ & $-2.04(0.20)^{* * *}$ \\
\hline Obese $(1=$ Yes; 0 = No $)$ & $-1.63(0.19)^{* * *}$ & $-1.32(0.19)^{* * *}$ \\
\hline Less than HS $(1=$ Yes; $0=$ No $)$ & & $-1.32(0.26)^{* * *}$ \\
\hline Some college $(1=$ Yes; $0=$ No $)$ & & $1.53(0.22)^{* * *}$ \\
\hline College/more $(1=$ Yes; 0 = No $)$ & & $2.80(0.22)^{* * *}$ \\
\hline Survey $(1992=1 ; 2004=0)$ & $2.01(0.18)^{* * *}$ & $2.67(0.18)^{* * *}$ \\
\hline $\mathbf{R}^{2}$ & 0.82 & 0.83 \\
\hline
\end{tabular}

Note: Pooled HRS 1992 and 2004 samples excluding inconsistent responses -Individuals aged 50-61.

$N=18,652$, Clusters $=9,326$.

Due to changes in the questions, the inconsistent responses for the 1992 HRS are those that satisfy $\mathrm{P}(75) \leq \mathrm{P}(85)$, and the inconsistent responses for 2004 HRS are those that satisfy $\mathrm{P}(75) \leq \mathrm{P}(80)$.

${ }^{\dagger} \mathrm{p}<.1,{ }^{*} \mathrm{p}<.05,{ }^{* *} \mathrm{p}<.01 ;{ }^{* * *} \mathrm{p}<.001$ (two-tailed tests).

mortality. This result is important because it suggests that empirical results based on complete samples are likely to be flawed.

Are estimates equally sensitive to the treatment of focal responses? Although we cannot entirely dismiss focal responses centered on zero (highly pessimistic assessment) or on one (highly optimistic assessment), it appears to be the case that respondents choose these values as a simplified form of expressing the direction of their expectations. Clearly, the integrated force of mortality is neither infinite (pessimistic assessment) nor zero (optimistic assessment). Likewise, it may be the case that some of the individuals who provided focal responses centered on 0.50 used this value as a device to express "neutrality," and were unaware that their choice implied mortality rates five to 10 times higher than the observed rates. Thus, using focal responses as if they were outcomes of individual computations distorts the intentionality of the responses. Nor can we treat the issue of focal responses as a kind of missing value problem, and then deploy the standard machinery of multiple imputation. Careful researchers attempt to "redistribute" focal responses using various methods, particularly the 0.50 responses (Bassett and Lumsdaine; Bruine de Bruin et al. 2002; Gan et al. 2005; Lumsdaine and van Loon 2012; Manski and Molinari, 2010). The problem is that these methods usually rely on strong, unverifiable assumptions, and leave open the possibility that reallocation could lead to worse distortions than the naïve avoidance of the problem. In the absence of a clear-cut strategy, we opt to compute the boundaries of uncertainty after imposing alternative constraints on the sample used for estimation (see Tables A.1, A.2, A.3, 


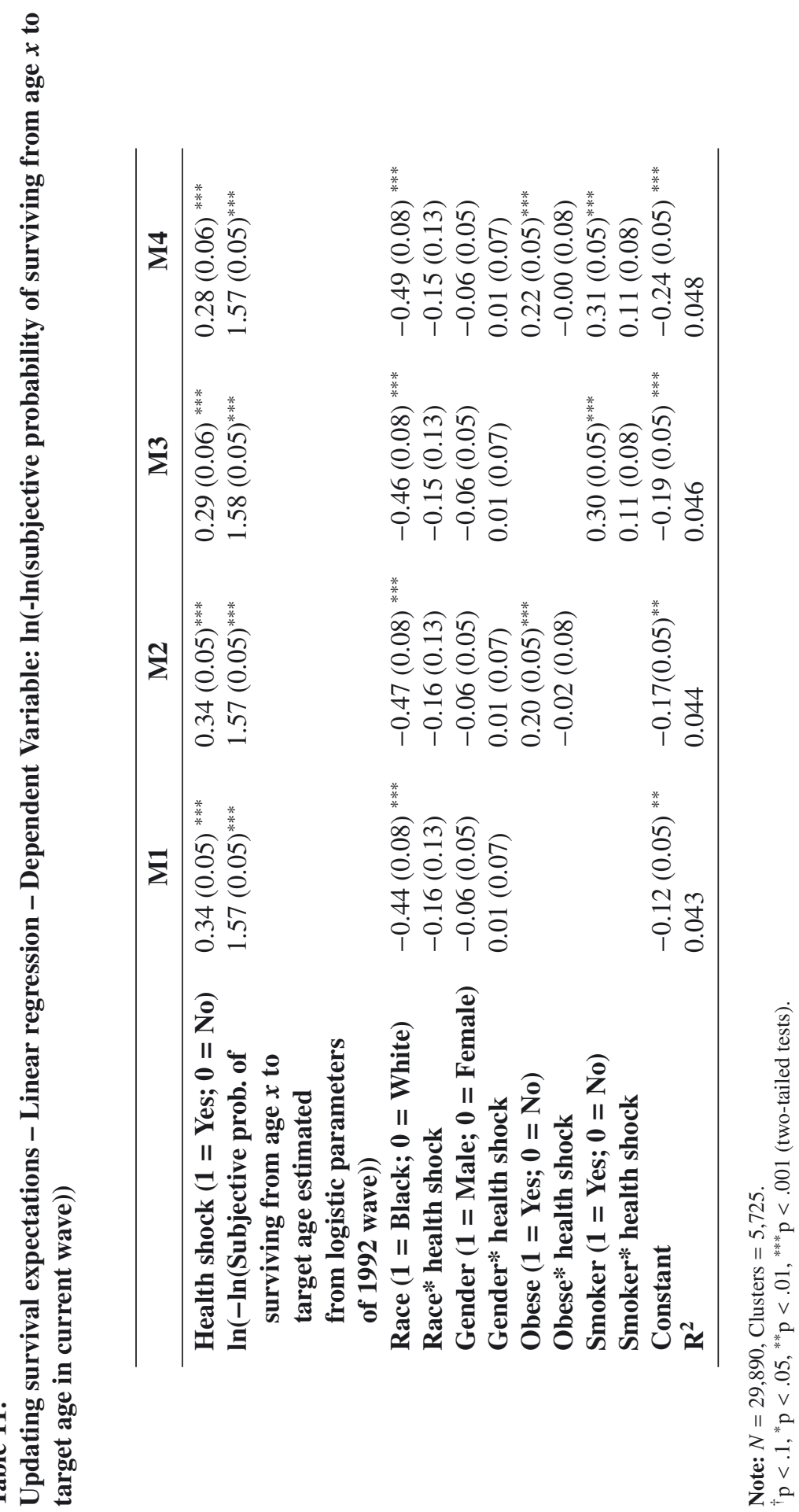


A.4, A.5 and A.6 in the appendix). These results suggest that the sensitivity of the parameter estimates to the treatment of focal responses is model-dependent. In general, we find that the estimates of parameters are more sensitive to the exclusion of the $50 \%$ response.

To summarize, we find that the patterns of mortality estimated from subjective survival expectations reflect quite accurately the patterns of observed or objective mortality. These subjective expectations deviate from observed patterns to the same extent as independent assessments of objectives mortality, such as those from HRS data and from U.S. life tables, differ from each other. Our results suggest that white men and women of both races tend to underestimate their survival chances, and that black men tend to greatly overestimate their survival chances.

We have argued that extreme care must be exercised with samples that include inconsistent responses, and that the best strategy is to exclude them altogether. Not unexpectedly, we find that alternative treatments of focal responses produce different results, but that the levels of uncertainty surrounding their use are modest. Thus, we suggest that future work on subjective expectations place more emphasis on defining a range for the parameters of interest (instead of point estimates with standard deviations), rather than on the identification of methods for allocating focal responses to "proper" numerical values, which is a rather risky enterprise. This strategy is also consistent with the idea of explicitly accounting for individual uncertainty regarding individual expectations (Willis, 2005).

\section{Fine-tuned models for subjective expectations}

Do the mortality risks implied by subjective survival expectations behave consistently in more refined analyses? For example, are the expectations of individuals with different behaviors and/or health statuses consistent with objective experiences associated with groups exhibiting the same behaviors or health statuses? Do individuals update their expectations in accordance with past experience; e.g., are they sensitive to individual exogenous shocks? Do younger birth cohorts adjust their expectations to reflect mortality improvements at older ages? To examine these questions, we propose fine-tuning the models formulated above to incorporate covariates.

We showed before that a logistic model fitted to the conditional subjective survival expectations $S_{x}(75)$ and $S_{x}(85)$ yields estimates of the (unconditional) survival curve that are quite close to both the survival curve that describes the observed HRS and the U.S. mortality experiences during 1992-2006. We now extend the logistic model to include the effects of covariates. To estimate these models, we assume that individual traits and behaviors modify the level parameter of the logistic function, while the ancillary parameter is invariant throughout. ${ }^{12}$

12 We thus follow standard practice in the estimation of hazard models. 
The "level" parameter reflects the average (underlying) mortality risk, whereas the ancillary parameter reflects the age pattern of mortality risks. The conditional probability of surviving to age $y$ given the respondent's age is $x$ is defined as:

$$
S_{x}(y)=\left(1+\exp \left(\left(y-\eta\left(g\left(Z_{i}\right)\right)\right) / \tau\right)\right) /\left(1+\exp \left(\left(x-\eta\left(g\left(Z_{i}\right)\right)\right) / \tau\right)\right)
$$

where $y$ is either 75 or $85, x<y, Z_{i}$ is a vector of characteristics, and $g($.$) is$ a functional form that, for simplicity, we define as linear. The hazard function corresponding to this conditional survival is:

$$
\mu(v)=(1 / \tau) * \exp \left(\left(\left(v-\eta\left(g\left(Z_{i}\right)\right)\right) / \tau\right)\right) /\left(1+\exp \left(\left(x-\eta\left(g\left(Z_{i}\right)\right)\right) / \tau\right)\right.
$$

We pool together the initial HRS cohort from 1992 with the HRS refreshment cohort from 2004, and create a dummy variable to distinguish them. This will help us compare two birth cohorts with different experiences. In the younger cohort, the proportions of whites, women, and individuals with at least some college education are statistically larger. Moreover, and as expected, the 1992 cohort includes a statistically larger proportion of current smokers than the 2004 cohort, whereas the prevalence of obesity is significantly higher in the younger than in the older cohort (see Tables 1 and 2). We then use the pooled sample to estimate the effects of the covariates on the parameters of model (1) using the (elicited) survival expectations to age 75 and to age 85 (or 80 ). ${ }^{13}$ The covariates include binary variables for gender, race, HRS wave (1992 versus 2004), age (50-55 versus 56-61), and several interactions.

\subsection{The effects of traits and behaviors}

Table 9 displays the estimates of five models and the corresponding estimates associated with individual characteristics. Because of scaling, a negative coefficient for a covariate implies higher mortality risks. Model 1 shows statistically significant differences by race and gender: blacks and men had survival expectations reflecting higher mortality risks than whites and women, respectively. Model 2 reveals that the subjective expectations of the 1992 HRS cohort (column 2) implied lower mortality risks than the subjective expectations of the 2004 refreshment cohort. In the same model, the effects of race are downgraded to statistical insignificance. Models 3 and 4 show that the younger HRS cohort had subjective expectations that reflected a mortality pattern with lower mortality risks than those of the older HRS cohort. These effects are large and statistically significant. Finally, Model 5 includes

\footnotetext{
13 Estimates of the parameters' standard errors are estimated using clustered corrections since each individual contributes two observations corresponding to the two conditional probabilities $\mathrm{Sx}(75)$ and $\mathrm{Sx}(85$ or 80$)$. Estimates were obtained from the restricted samples; that is, from the samples that excluded inconsistent answers but included focal answers.
} 
interaction effects, none of which are found to be strongly ( $p<.01$ or even $\mathrm{p}<.05$ ) statistically significant.

Are the effects in Table 9 large enough to matter at all? It can be shown (see the appendix) that an absolute change equal to $\delta$ in the value of the logistic scale parameter implies an approximate change of about $-0.0062^{*} \delta$ in the force of mortality around age 70 . Thus, for example, the largest coefficient associated with membership in the 1992 cohort (2.01) translates into a mortality risk at around age 70 that is 0.0124 lower than the mortality risk associated with membership in the refreshment cohorts. A difference of this magnitude is equivalent to an upward shift in life expectancy at age 50 of about three years, or about 10 percent of the actual U.S. life expectancy at age 50. The differences associated with race and gender are, respectively, one-tenth and two-thirds as large as the effects associated with cohort membership, whereas the differences associated with age group are about 0.42 as large.

We wish to highlight two findings. Why should the individuals in the younger age group express subjective expectations that imply lower mortality than the individuals in the older age group? The younger individuals' implied life expectancies at age 50 were about 1.3 years higher than those of the older individuals. This result contradicts findings from research using different datasets (Wu et al. 2014). However, since these results were compromised because inferences were drawn using separate responses about different target ages - a practice that we believe should be discouraged - we should downplay the importance of these findings. More significantly, however, this result is inconsistent with the literature on self-reported health status showing that older people tend to report being in better health than their younger counterparts (Jylhä et al. 2001). Unlike models in the literature on health self-reports, our models do not control for indicators of individual health conditions or disability. Thus, this inconsistency could have arisen because the average objective health status among older individuals was worse than the average objective health status among younger individuals. However, this was not shown to be the case. In fact, when we estimated our models while controlling for both self-reported ADL and self-reported chronic conditions, we found that the coefficient for the age dummy continued to be statistically significant and pointed toward a more pessimistic assessment among the older than among the younger age group (see Table 9).

Since the finding is unlikely to be due to cohort effects (see below), we are left with the possibility that subjective survival expectation assessments and selfreported health among older individuals tap into very different dimensions than among younger individuals. ${ }^{14}$

14 The finding reported here is based on broad, coarse age groups. We chose this approach because we wanted to roughly follow the strategy employed in the extant literature, and not because it is the best strategy available. Thus, while it may be possible to explore different functional forms for age (discrete and continuous), such an approach could flounder because the estimation becomes increasingly fragile as the classification of age groups becomes more fine-grained. 
The second noteworthy finding is that members of the original HRS cohort had survival expectations that implied mortality risks associated with higher life expectancy at age 50. The differences are equivalent to shifts of about three years less than in the 2004 refreshment cohort. Why should this be the case? Are the differences between the cohorts in Table 9 due to differences in the prevalence of traits that we are not accounting for?

Table 10 displays estimates of a model that includes controls for additional covariates that could influence survival expectations: namely, education, obesity, and smoking status (current, ever, and never). The inclusion of new control variables does not remove the effects under scrutiny, and the 1992 cohort continued to express subjective expectations implying a more forgiving survival curve. In fact, judging by the magnitude of the coefficient (2.67), it appears that the benefits are even greater than those revealed by Table 9 .

The results in Table 10 also demonstrate two additional associations of interest. First, the effects of smoking and obesity status are statistically significant, are properly signed, and are very large. Indeed, the smokers' survival expectations translate into mortality risks at age 70 that are about $0.0167(2.69 * 0.0062)$ higher than those of the non-smokers, a value that differs almost $36 \%$ from the average mortality in the population at that age. This finding is nothing short of remarkable, as the estimated observed mortality excess at ages 75-79 due to smoking in the U.S. is close to 25\% (National Research Council 2011) The difference is equivalent to smoking-related losses in life expectancy at age 50 of about 3.73 years, or $15 \%$ of observed U.S. life expectancy at age 50.

The effects of obesity are more modest, but are still quite large, at about twothirds the size of the effects due to smoking. These findings are in line with rough estimates of mortality excess due to obesity at ages 50 and older (Ho and Preston 2010).

The second interesting result in Table 10 is the education gradient of subjective survival: the sign of the estimated slope not only reproduces the sign of the observed education mortality slope in the U.S., but its magnitude is close to that of estimates for the U.S. in 2000. The difference in survival between those with a college education or more and those with less than a high school education is equivalent to about three years of life expectancy at age 50 (Palloni and Thomas 2013), whereas Table 10 implies a difference of about 1.4 years. ${ }^{15}$ Once again, these differences are not larger than the differences routinely found between alternative estimates from observed data.

15 The U.S. life table for the college-educated has a life expectancy at age 50 of about 31 years, whereas the life table for individuals with less than a high school education is about 28 years. From Table 10: the estimated difference in the mortality levels between those at the extremes of the education distribution is close to one. This implies a shift in mortality risks of about 0.0062 (see the appendix), which in turn translates into a differences in life expectancy at age 50 of about 1.4 years. 


\subsection{The effects of shocks: updating survival expectations}

With the exception of the cohort effects identified above, subjective and objective survival are in sync with each other. We could also conjecture that individuals not only appraise their survival chances with a relatively high degree of accuracy, but that if they do so, they adjust their appraisals in response to external events. If this is the case, then individual expectations should be subject to significant variability owing to the ebb and flow of exogenous shocks, particularly those that are healthrelated. To test this conjecture, we adopt a model suitable for estimating the effects of time-dependent events (shocks) on time-dependent survival expectations. The models estimated previously are useful when all of the covariates are fixed, but are cumbersome to estimate and interpret when one or more of these covariates vary over time (including the time-varying effects of a fixed covariate).

We introduce a simplifying assumption that preserves the idea that survival expectations mirror underlying mortality risks that are logistic, but that simultaneously enables us to assess the effects of time-dependent external shocks. We propose the following model:

$$
S_{i x}(t, x+k)=\left[S_{x}^{s}(x+k)\right]^{g(Z i)}
$$

where $S_{i x}(t, x+k)$ is the expected conditional probability of surviving from age $x$ to age $x+k$ elicited at time $t$ by individual $i$ who is endowed with a vector of relevant individual characteristics $Z_{i}$. This is the standard expression for the survival function of a proportional hazard model where the standard is unknown, and can either be neglected (Cox models) or estimated directly (parametric hazard models). The function $S_{x}^{s}(x+k)$ is a standard (reference) survival function, and $g($.$) is a$ function that translates the vector of characteristics $Z_{i}$ into changes in the mortality risks underlying the survival function. We choose as a standard the logistic survival function estimated from the initial HRS cohort in 1992, assume that $g($.$) is a linear$ function, and take logs on both sides to obtain

$$
\ln \left(-\ln \left(S_{i x}(t, x+k)\right)=\beta Z_{i}+\ln \left(\ln S_{x}^{S}(x+k)\right)\right.
$$

We choose to identify the standard ex ante based on the assumption that we already have sufficient information (contained in the standard) to capture the role of aging alone in the formation of survival expectations. What this information leaves out is precisely what we are interested in: namely, the effects on the levels of expected mortality produced by exogenous health shocks. These effects will be reflected as shifts in estimates of the beta coefficient.

A few caveats are in order. First, a covariate with a positive effect in these models shifts mortality risks upward, and flattens the survival curve. Second, if the model is correct, then the estimate of the slope relative to the double log of the standard survival function should be one. Departures of the slope of the regression line from unity suggest either non-proportional hazards or shifts in the standard mortality patterns embedded in survival expectations. In either case, we can use departures 
from one as a measure of the realism or the suitability of the model. Third, because we need to have full information about the events unfolding over a long period of time, we focus only on the original 1992 HRS cohort, and follow the trajectory of individual responses regarding wave-specific subjective survival. Finally, the vector $Z$ contains control variables (education, gender, race, smoking, and obesity status) and a time-dependent dummy variable that flags whether the individual experiences a health shock in the preceding interwave periods. ${ }^{16}$ For simplicity, we only use a one-wave lag specification; e.g., the survival expectations formed at period $t$ depend on health events that occurred in the interwave period immediately preceding $t$.

Table 11 shows the most important results. First, the estimate of the slope coefficient is slightly larger than one. This implies that the individuals may be using a mortality pattern that rises more steeply with age than the standard logistic we adopt here. Second, the interwave individual health shocks have effects that are properly signed, are statistically significant, and are quite large. Indeed, an estimated effect of 0.34 in Table 11 translates into a decrease in life expectancy at age 50 of about 1.5 years: that is, individuals who experienced at least one health shock in the preceding interwave period adjusted their mortality expectations by adopting a survival function that implies 1.5 fewer years in residual life expectancy at age 50 . Note that these are lower bound effects, since (a) they do not take into account the possibility that a particular health shock could have effects spread across a number of time periods, and not just one; and (b) we do not distinguish between types of health shocks, and therefore assume that their effects are homogeneous, which is a somewhat unrealistic assumption.

Finally, obesity and smoking behave as would be expected: obese individuals and particularly smokers adjusted their survival expectations downward upon experiencing a health shock (the interaction effects of smoking and health shock are positive and significant) to a greater extent than their non-obese or non-smoking peers.

\section{Discussion}

We highlight two sets of findings, substantive and methodological. The substantive findings can be summarized as follows:

1. The subjective probabilities are quite close to the estimates in the actual life tables, and they preserve the basic observed differentials across subgroups.

2. Whites are more "pessimistic" (their expected survival underestimates observed survival) than blacks, and women are more pessimistic than men.

16 We define as a health shock a diagnosis of the presence of health conditions that were not present in prior waves, including heart disease, diabetes, cancer, lung diseases, and stroke. 
3. There is a large but unexplained difference between individuals in the oldest and in the youngest age groups, as those in the former group underestimate their survival chances more than those in the latter group.

4. There is a surprising and unexplained contrast between the original and the more recent cohorts in the HRS (1992 versus 2004). This contrast does not vanish after controlling for important covariates.

5. The objective, observed differentials between smokers and non-smokers, obese and non-obese individuals, and highly educated and less educated individuals are reflected very well in the subjective expectations of survival.

6. There are very large updating effects that become stronger among individuals exposed to higher risks.

By and large, our results are in agreement with those of the recent literature (Roebuck Bulanda and Zhang 2009; Irby-Shasanmi 2013; Zick et al. 2014). Our model fits as well or better than previous models (Hudomiet and Willis 2012; Ludwig and Zimper 2013); and, in contrast to other studies (Elder 2013), we find that subjective forecasts perform quite well, especially when applied to small subpopulations characterized by particular behaviors (smoking) or traits (obesity, education). Finally, we find that younger individuals tend to be more optimistic that older individuals, and that these differences are not attributable to changes in objective health status.

There are three methodological results of note. First, analyses of subjective expectations should not include inconsistent responses, since they lead to flawed estimates. Although the study of inconsistent responses is a legitimate activity on its own, including these responses in analyses involving comparisons with observed survival will lead the research astray. This note of caution applies to studies that utilize multiple subjective expectations, which usually refer to different ages, as if they were unrelated to each other. Indeed, we believe that findings such as those of Elder (2013) and Hammermesch (1985), which show that there is a "tendency of individuals to understate the likelihood of living to relatively young ages while overstating the likelihood of living to ages beyond 80" (Elder 2013), are artifacts of the data that the exclusion of inconsistent responses would eliminate.

Second, the same note of caution is not applicable to focal responses, since they do not have a large influence on the results. This is demonstrated by our finding that alternative treatments lead to similar results, at least in the HRS and with the class of models we estimated.

Third, we recommend the use of relational models to estimate the effects of multiple covariates. A relational model approach allows for crisp interpretations; can be estimated very easily; offers a platform for rigorous comparisons across disparate datasets; and, finally, provides additional tools for verifying the plausibility and fit of the model to the data.

There is still much work to be done in this area. There are, for example, a host of unexplored relationships involving subjective survival expectations and strategic behaviors like savings, retirement, bequests, exposure to risks, and compliance with 
medical treatment; all of which could influence policies and programmatic health agendas. For example, why do individuals use subjective survival expectations to make decisions about bequests, labor force participation, and retirement? What is the ripple effect of an exogenous health shock on expectations? Does updating actually translate into behavioral changes, such as adopting a new diet or engaging in more exercise? How do individuals parse information about advances in medical technologies and new discoveries, and how is this information incorporated into their survival expectations? For example, could optimism regarding possible advances in screening and therapies lead individuals to abandon vigilant behavior to such an extent that potential mortality improvements are undermined? Ultimately, if subjective expectations accurately reflect objective mortality conditions, they could be used as complementary indicators when studying SES differentials. The advantage of using this approach is that the data on subjective expectations provide much more detail than is available from the standard sources that are routinely used for the study of mortality differentials.

Finally, understanding the process of the formation of subjective survival expectations could shed light on health and mortality differentials across societies and cultures. Group differentials in the mechanisms that shape individual expectations about mortality risks may tell us a great deal about the pathways that lead to the production of objective heath and mortality differentials.

\section{References}

Bassett, W. F. and R. L. Lumsdaine 2001. Probability limits: Are subjective assessments adequately accurate? The Journal of Human Resources 36(2): 327-366.

Benítez-Silva, H. and H. Ni 2008. Health status and health dynamics in an empirical model of expected longevity. Journal of Health Economics 27(3): 564-584.

Bruine de Bruin, W., P. S. Fischbeck, N. A. Stiber, and B. Fischhoff 2002. What number is "Fifty-Fifty"?: Redistributing excessive 50\% responses in elicited probabilities. Risk Analysis 22(4): 713-723.

Elder, T. E. 2007. Subjective Survival Probabilities in the Health and Retirement Study: Systematic Biases and Predictive Validity. MRRC Working Paper WP 2007-159. Michigan Retirement Research Center (MRRC). University of Michigan.

Elder, T. E. 2013. The predictive validity of subjective mortality expectations: evidence from the health and retirement study. Demography 50: 569-589.

Falba, T. A. and S. H. Busch 2005. Survival expectations of the obese: Is excess mortality reflected in perceptions? Obesity Research 13(4): 754-761.

Gan, L., M. D. Hurd, and D. L. McFadden 2005. Individual subjective survival curves. In Analyses in the economics of aging, ed. D A. Wise, 377-402. Chicago: University of Chicago Press.

Hamermesh, D. S. 1985. Expectations, life expectancy, and economic behavior. The Quarterly Journal of Economics 100(2): 389-408.

Ho, J. Y. and S. H. Preston 2010. U.S. mortality in an international context: Age variations. Population and Development Review 36(4): 749-773. 
Horiuchi, S. and J. R. Wilmoth 1998. Deceleration in the age pattern of mortality at older ages. Demography 35(4): 391-412.

Hudomiet, P. and R. J. Willis 2012. Estimating Second Order Probability Beliefs from Subjective Survival Data. NBER Working Paper 18258. National Bureau of Economic Research (NBER), Cambridge, MA.

Hurd, M. D., D. McFadden and A. Merrill 1999. Predictors of Mortality among the Elderly. NBER Working Paper 7440. National Bureau of Economic Research (NBER), Cambridge, MA.

Hurd, M. D. and K. McGarry 1995. Evaluation of the subjective probabilities of survival in the health and retirement study. Journal of Human Resources 30 (0 Special Issue on the Health and Retirement Study: Data Quality and Early Results): S268-S292.

Hurd, M. D. and K. McGarry 2002. The predictive validity of subjective probabilities of survival. The Economic Journal 112: 996-985.

Idler, E. L. and Y. Benyamini 1997. Self-rated health and mortality: A review of twenty-seven community studies. Journal of Health and Social Behavior 38: 21-37.

Irby-Shasanmi, A. 2013. Predictors of subjective life expectancy among African American. Research on Aging 35: 322-347.

Jylhä, M., J. M. Guralnik, J. Balfour and L. P. Fried 2001. Walking difficulty, walking speed, and age as predictors of self-rated health. Journal of Gerontology: Medical Sciences 56A(10): M609-M617.

Juster, F. T. and R. Suzman 1995. An overview of the health and retirement study. Journal of Human Resources 3 (Special Issue on the Health and Retirement Study: Data Quality and Early Results): S7-S56.

Kannisto, V., J. Lauritsen, A. R. Thatcher and J. W. Vaupel 1994. Reductions in mortality at advanced ages: Several decades of evidence from 27 countries. Population and Development Review 20: 793-870.

Khwaja, A., F. Sloan and S. Chung 2006. Learning about individual risk and the decision to smoke. International Journal of Industrial Organization 24: 683-699.

Khwaja, A., F. Sloan, and S. Chung 2007. The relationship between individual expectations and behaviors: mortality expectations and smoking decisions. Journal of Risk and Uncertainty 25: 179-201.

Lillard, L. and R. Willis 2001. Cognition and Wealth: The Importance of Probabilistic Thinking. MRRC Working Paper WP 2001-007. University of Michigan Retirement Research Center (MRRC), Ann Arbor, MI.

Liu, J.-T., M.-W. Tsou and J. Hammit. 2007. Health Information and Subjective Survival Probability: Evidence from Taiwan". NBER Working Paper 12864. NBER Working Paper Series. National Bureau of Economic Research (NBER), Cambridge, MA.

Ludwig, A. and A. Zimper 2012. A parsimonious model of subjective life expectancy. Theory and Decision 75(4): 519-541.

Lumsdaine, R. L. and R. J. D. Pooter van Loon 2012. Wall Street vs. Main Street: A Comparison of Views. NBER Working Paper 19103. NBER Working Paper Series. National Bureau of Economic Research (NBER), Cambridge, MA.

Manski, C. F. 2004. Measuring expectations. Econometrica 5(9): 1329-1376. 
Palloni, A. and J. R. Thomas 2013. Estimation of covariate effects with current status data and differential mortality. Demography 50: 521-544.

Peracchi, F. and V. Perotti 2010. Subjective Survival Probabilities and Life Tables: Evidence from Europe. Einaudi Institute for Economics and Finance (EIEF) Working Paper 10/16.

Perozek, M. 2008. Using subjective expectations to forecast longevity: Do survey respondents know something we don't know? Demography 45(1): 95-113.

Popham, F. and R. Mitchell 2007. Self-rated life expectancy and lifetime socio-economic position: Cross-sectional analysis of the British household panel survey. International Journal of Epidemiology 36: 58-65.

Post, T. and K. Hanewald 2010. Stochastic Mortality, Subjective Survival Expectations, and Individual Saving Behavior. Humboldt University, Berlin, Germany. SFB 649 Discussion Paper 2010-040.

Quesnel-Vallée, A. 2007. Self-rated health: Caught in the crossfire of the quest for 'true' health? International Journal of Epidemiology 36: 1161-1164.

Robine, J.-M. and J. W. Vaupel 2001. Supercentenarians: Slower ageing individuals or senile elderly? Experimental Gerontology 26(4): 915-930.

Roebuck Bulanda, J. and Z. Zhang 2009. Racial-ethnic differences in subjective survival expectations for the retirement years. Research on Aging 31(6): 688-709.

Scott-Sheldon, L. A. J., M. P. Carey, P. A. Vanable and T. E. Senn 2010. Subjective life expectancy and health behaviors among STD clinic patients. American Journal of Health Behavior 34(3): 349-361.

Siegel, M., E. H. Bradley and S. V. Kasl 2003. Self-rated life expectancy as a predictor of mortality: Evidence from the HRS and AHEAD Surveys. Gerontology 49: 265-271.

Singh-Manoux, A., A. Gueguen, P. Martikainen, J. Ferrie, M. Marmot, and M. Shipley 2007. Self-rated health and mortality: short- and long-term associations in the Whitehall II study. Psychosomatic Medicine 69: 138-143.

Smith, K. V., D. H. Taylor, F. A. Sloan, F. Reed Johnson and W. H. Desvousges 2001a. Do smokers respond to health shocks? The Review of Economics and Statistics 83(4): 675-687.

Smith, K. V., D. H. Taylor and F. A. Sloan 2001b. Longevity expectations and death: can people predict their own demise? The American Economic Review 91(4): 1126-1134.

van Doorn, C. and S. V. Kasl 1998. Can parental longevity and self-rated life expectancy predict mortality among older persons? Results from an Australian cohort. Journal of Gerontology 53B(1): S28-S34.

Willis, R. J. 2005. Comment on Gan et al. 2005. Individual subjective survival curves. In Analyses in the economics of aging, ed. D. A. Wise, 402-411. Chicago: University of Chicago Press.

Wu, S., R. Stevens and S. Thorp 2014. Die Young or Live Long: Modeling Subjective Survival Probabilities. Paper presented at the American Economic Association annual meeting held in Philadelphia, PA. January 3-5, 2014.

Young, H., E. Grundy, D. O'Reilly and P. Boyle 2010. Self-rated health and mortality in the UK: Results from the first comparative analysis of the England and Wales, Scotland, and Northern Ireland longitudinal studies. Office for National Statistics. Population Trends 139: 11-36. 
Zick, C. D., K. R. Smith, R. N. Mayer and L. B. Taylor 2014. Family, frailty, and fatal futures? own-health and family-health predictors of subjective life expectancy. Research on Aging 36(2): 244-266.

\section{Appendix: Relations between logistic survival function, hazard functions and residual life expectancies}

The expressions for the logistic unconditional probability of surviving to age $x$ and instantaneous mortality rate at age $x$ are

$$
\begin{aligned}
& S(x)=1 /(1+\exp (x-\tau) / \sigma) \\
& \mu(x)=(1 / \sigma)(1-S(x))
\end{aligned}
$$

The derivative of the mortality function with respect to $\tau$ is $\partial \mu(x) / \partial \tau=$ $-\left(1 / \sigma^{2}\right) S(x)(1-S(x))^{2}$. We choose an ancillary parameter equal to six, estimate the mortality rate at age 70 (approximately the middle of the range experienced by the original HRS cohort, $\mu(70)$ to be between 0.033 and 0.038 , and assign $S(70)$ a value close to that experienced in the U.S. in the year 2000 (between 0.75 and 0.82). We can then approximate the value of the derivative at around 0.0062. Computing the life expectancy at age 50 (the earliest age at which the original cohort was observed) and relating this to changes in $S(70)$ and $\mu(70)$ leads to the following relationship: a change of about 0.010 in mortality above age 50 to a change of about 2.31 years of residual life expectancy at age 50 .

Figure A.1:

Subjective survival to age 75 . Focal responses $(0,50$, and 100): Percentages by gender and race - HRS 2004

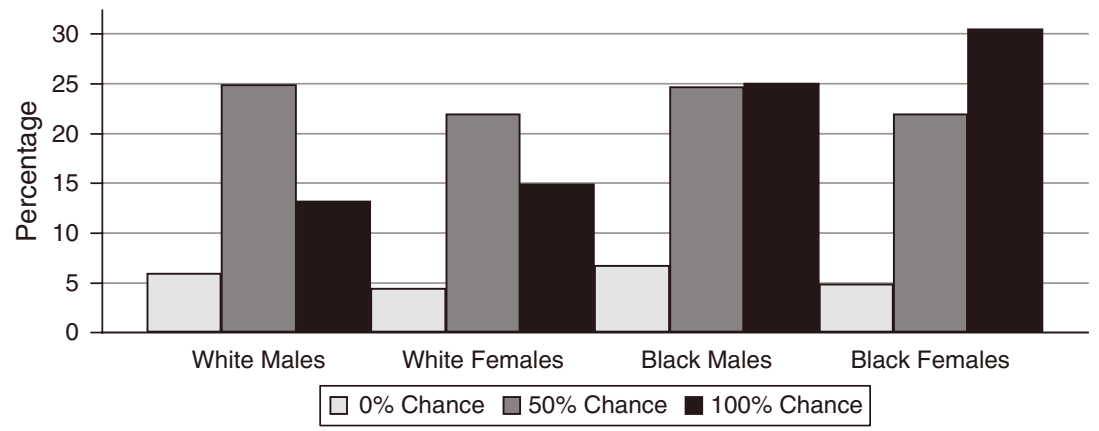


Figure A.2:

Subjective survival to age 80 . Focal responses $(0,50$, and 100): Percentages by gender and race - HRS 2004

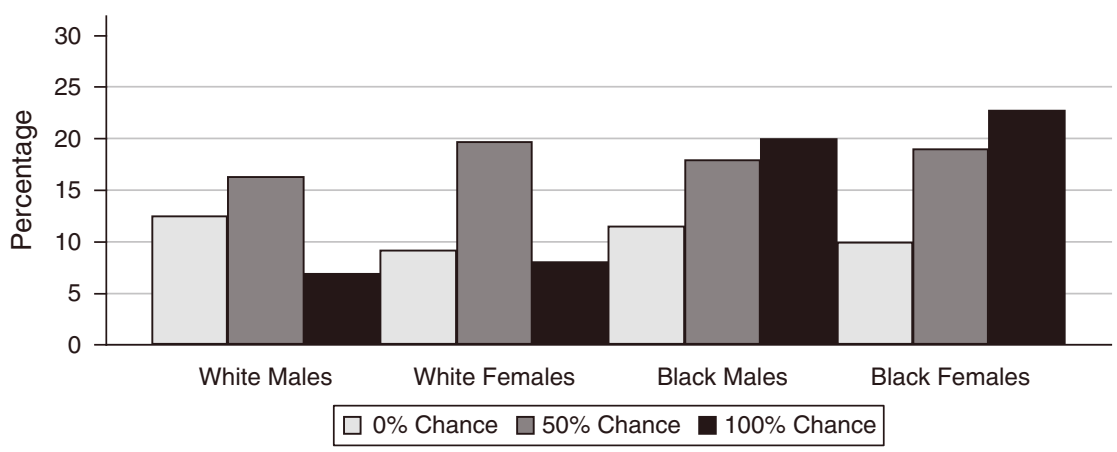

Figure A.3:

Subjective survival to ages 75 and 80 . Focal responses $(0,50$, and 100): Inconsistent responses percentages by gender and race - HRS 2004

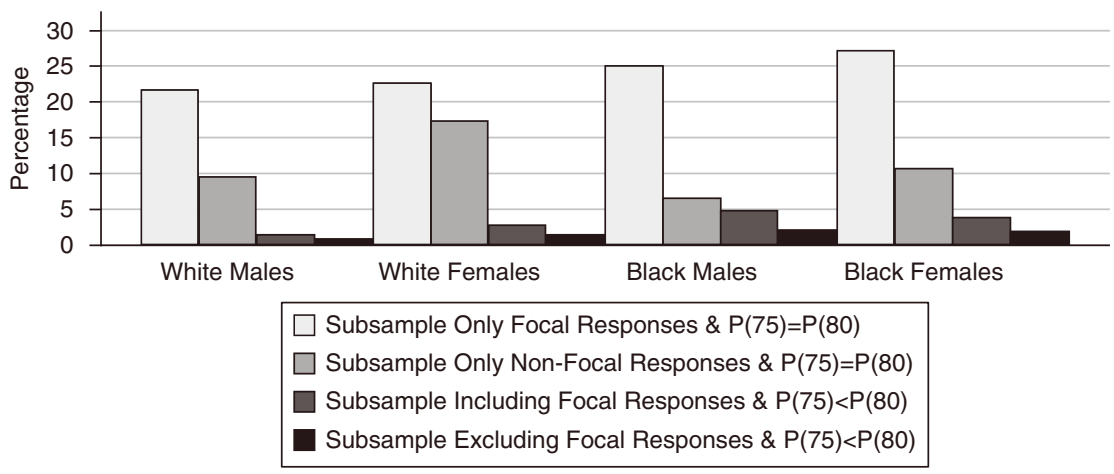




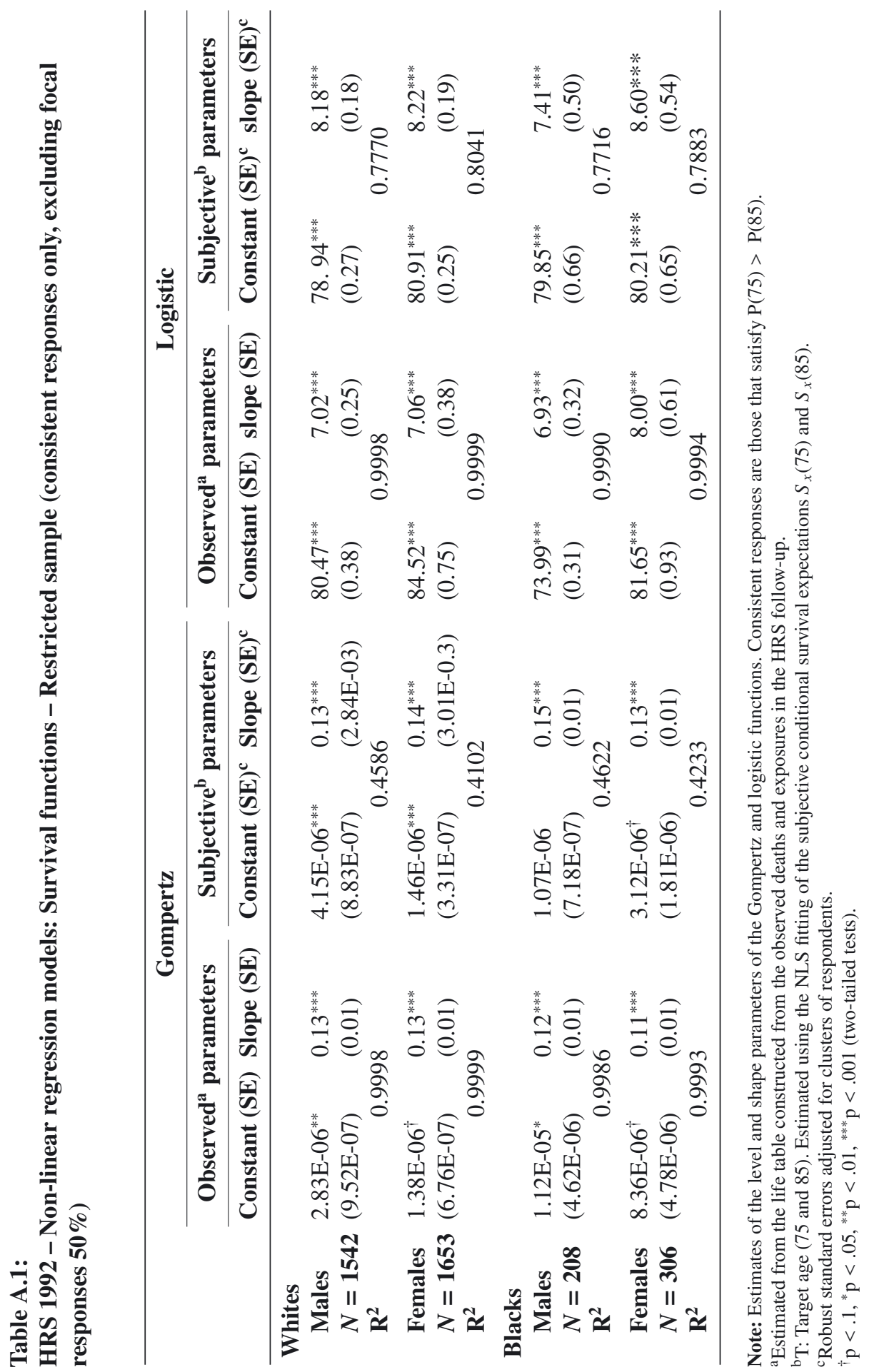




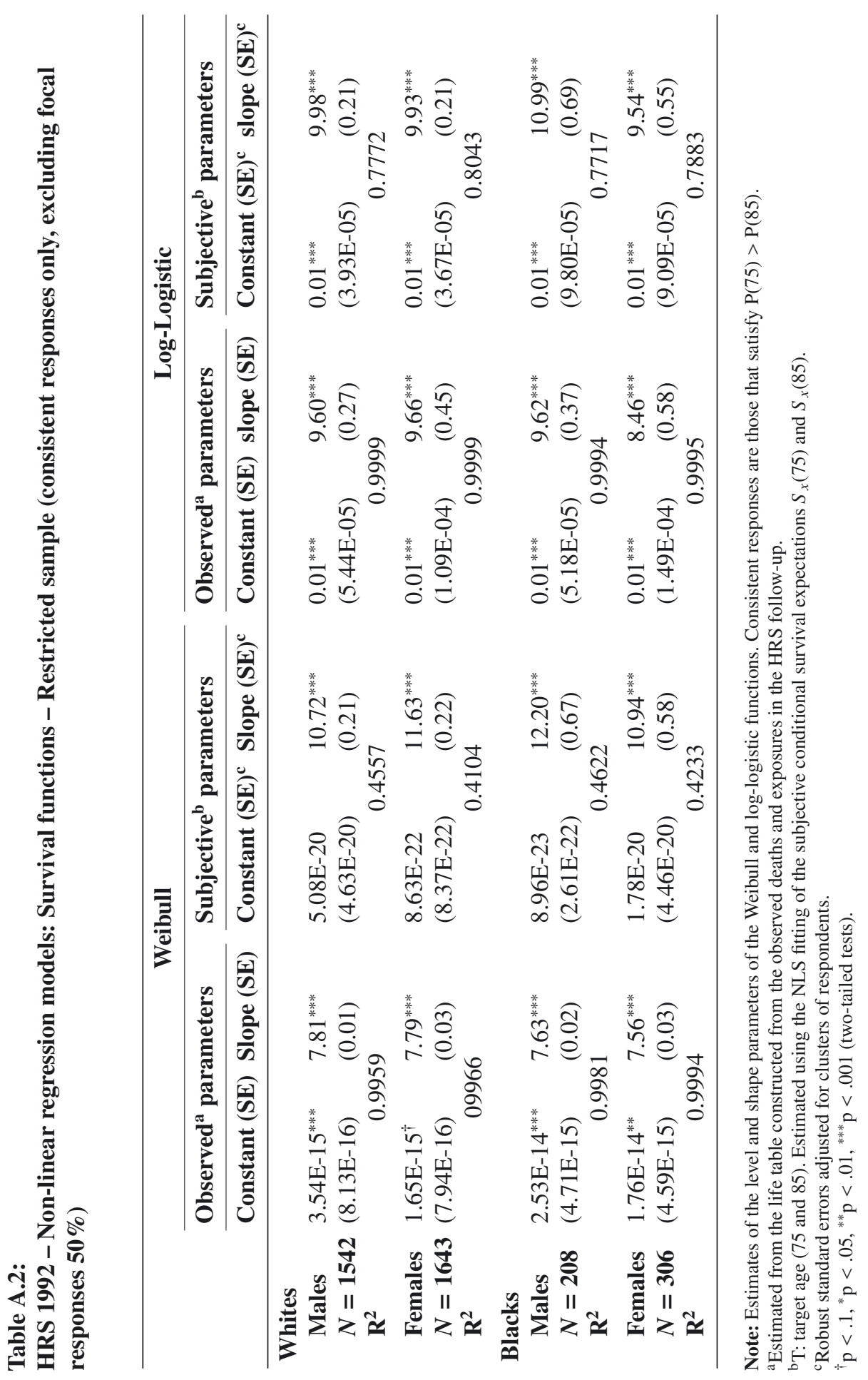




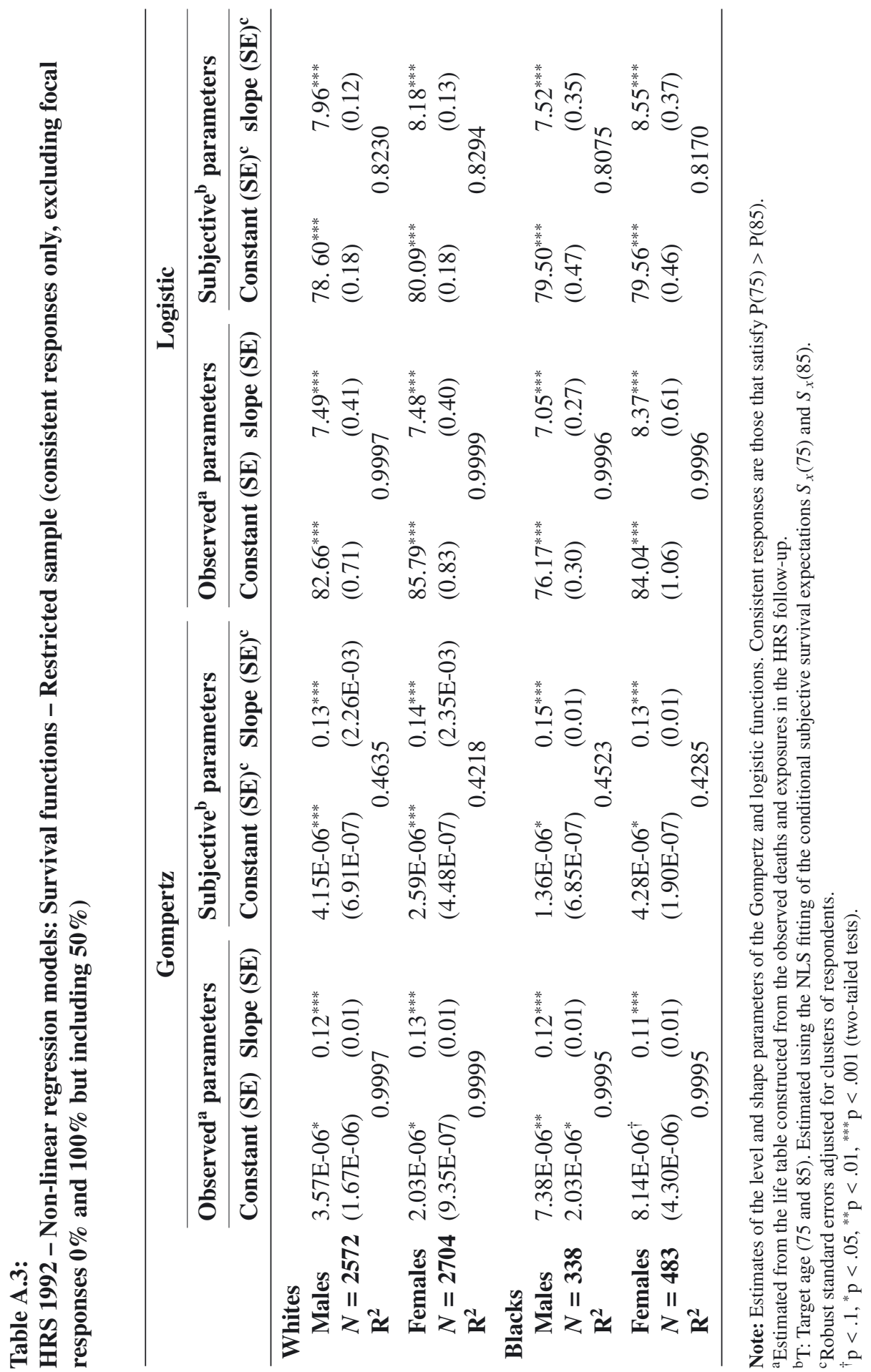




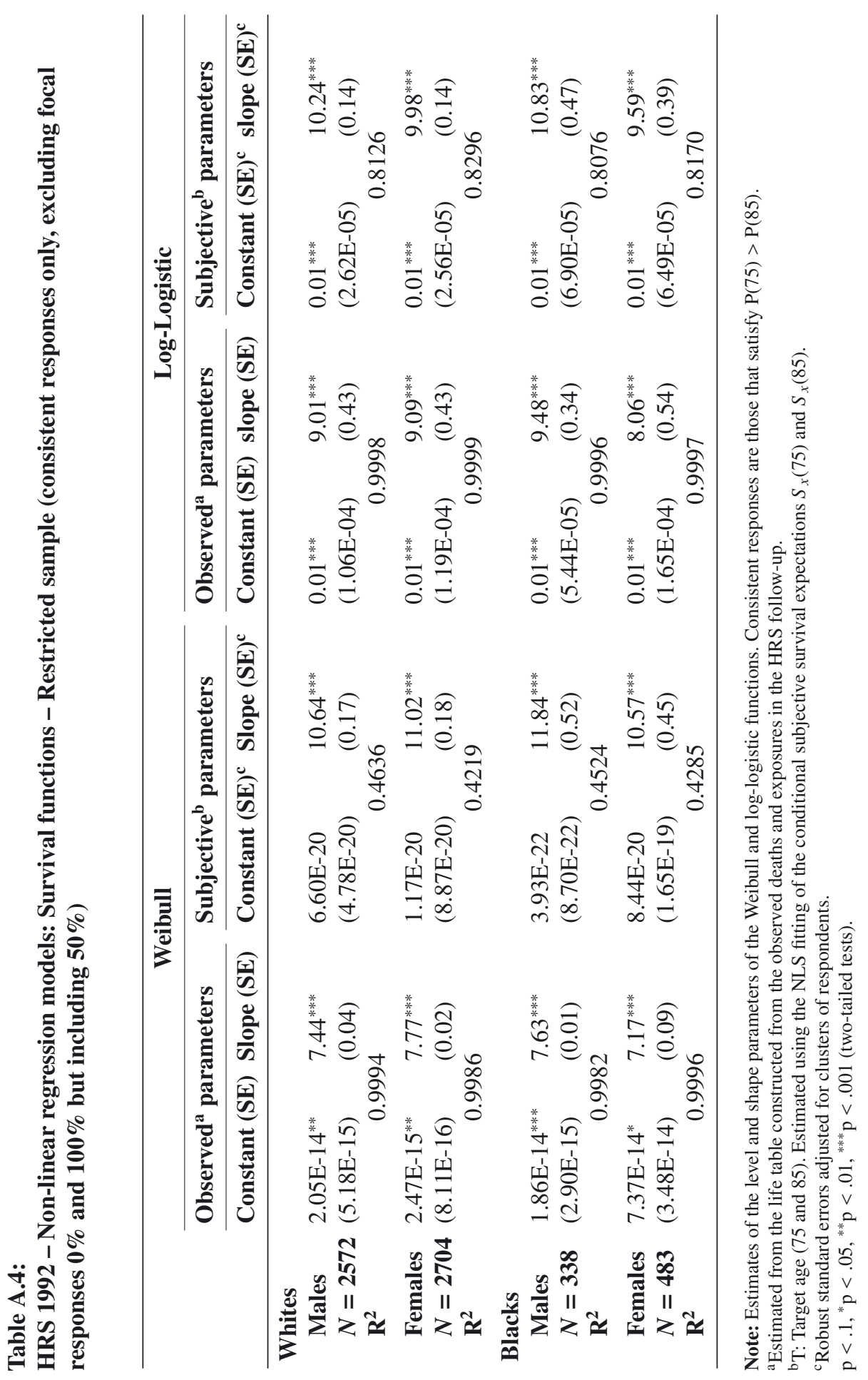




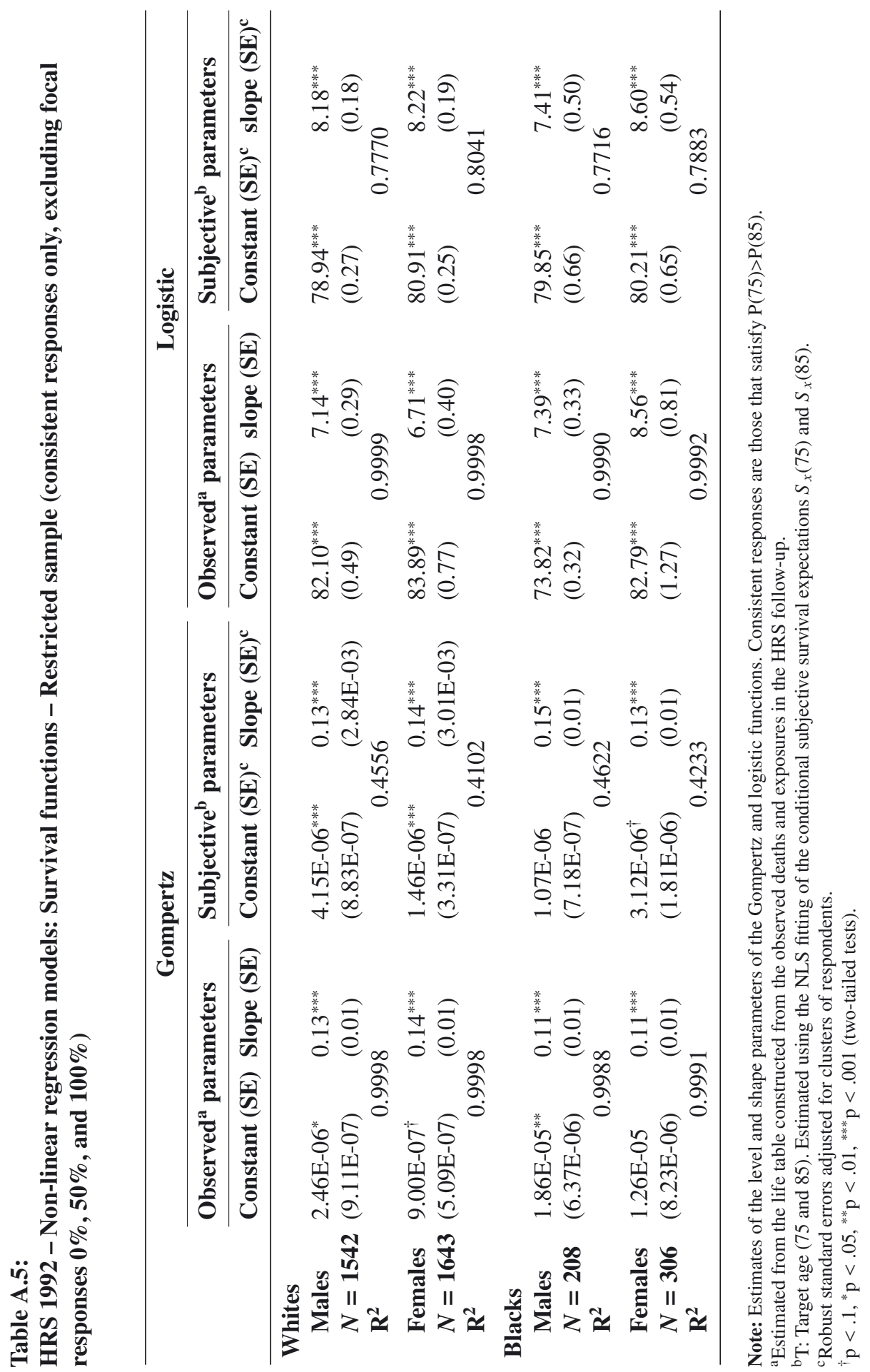




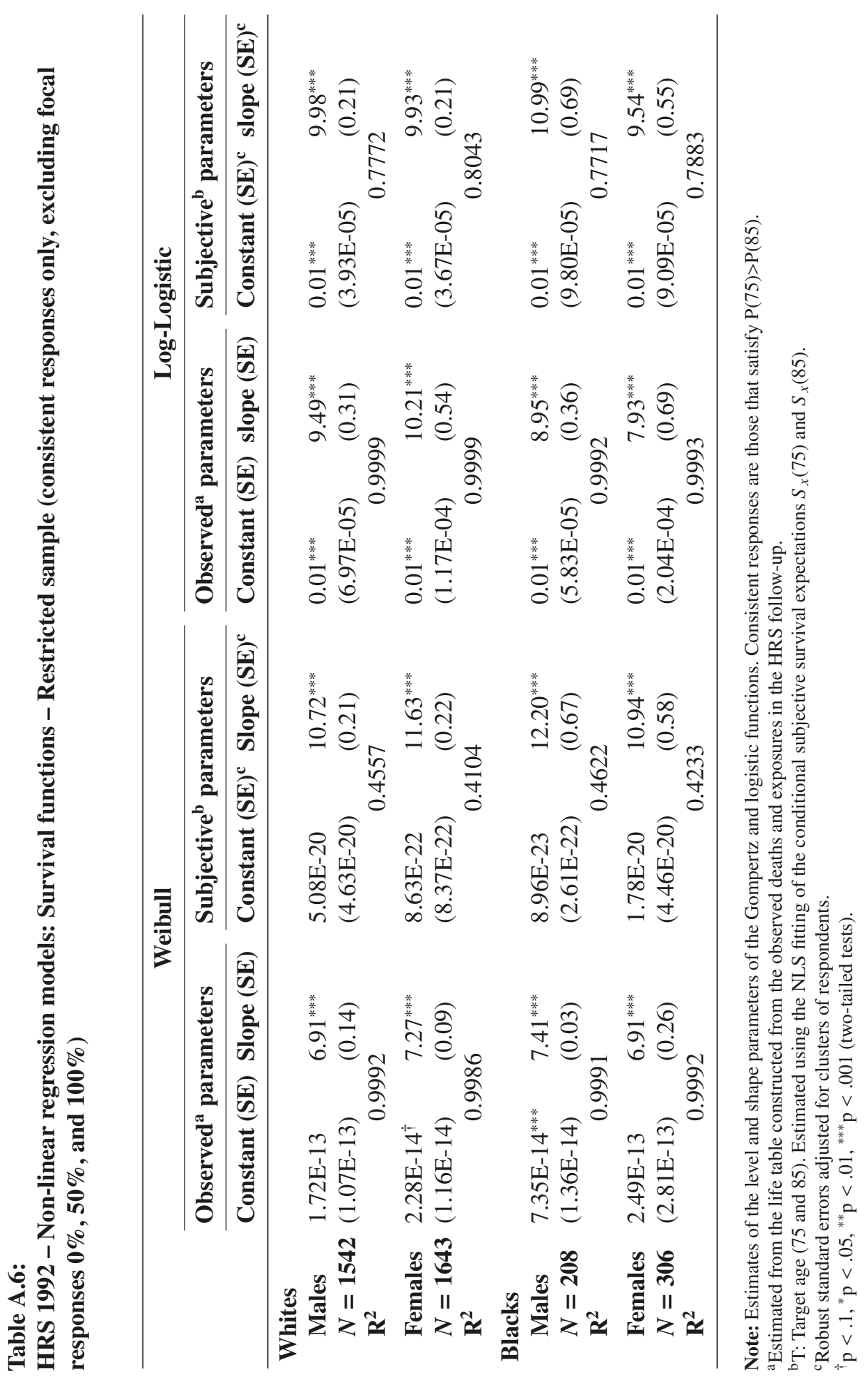


